\title{
Oak Ridge TNS Program: Technical Needs Assessment
}

\author{
W. R. Becraft \\ T. G. Brown \\ P. B. Mohr \\ Y-K. M. Peng \\ R. L. Reid \\ M. Roberts \\ R. Rosenfeld
}

\author{
C. Sardella \\ T E. Shannon \\ W. E. Simpson \\ P T. Spampinato \\ D. Steiner \\ W. M. Wells \\ G. W. Wiseman
}




\section{DISCLAIMER}

This report was prepared as an account of work sponsored by an agency of the United States Government. Neither the United States Government nor any agency Thereof, nor any of their employees, makes any warranty, express or implied, or assumes any legal liability or responsibility for the accuracy, completeness, or usefulness of any information, apparatus, product, or process disclosed, or represents that its use would not infringe privately owned rights. Reference herein to any specific commercial product, process, or service by trade name, trademark, manufacturer, or otherwise does not necessarily constitute or imply its endorsement, recommendation, or favoring by the United States Government or any agency thereof. The views and opinions of authors expressed herein do not necessarily state or reflect those of the United States Government or any agency thereof. 


\section{DISCLAIMER}

Portions of this document may be illegible in electronic image products. Images are produced from the best available original document. 


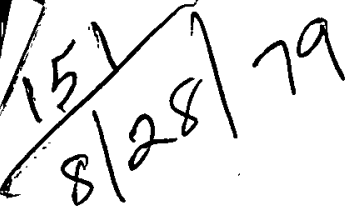

ORNL/TM-6722

Dist. Category UC-20 d

Contract No. W-7405-eng-26

FUSION ENERGY DIVISION

OAK RIDGE TNS PROGRAM: TECHNICAL NEEDS ASSESSMENT

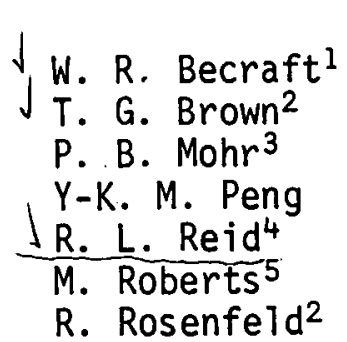

W. R. Becraft

P. B. Mohr ${ }^{3}$

Y-K. M. Peng

R. L. Reid

R. Rosenfeld $d^{2}$
C. Sardella 2
T. E. Shamon

W. E. Simpson ${ }^{2}$

P. T. Spanpinato 2

D. Steiner

W. M. We $11 \mathrm{~s}^{4}$

G. W. Wiseman ${ }^{2}$

${ }^{1}$ General Electric Company, Oak Ridge, Tennessee.

${ }^{2}$ Grumman Aerosdace Corporation, Bethpage, New York.

${ }^{3}$ Lawrence Livermore Laboratory, Livermore, California.

${ }^{4} U C C-N D$ Engineering.

50 ffice of Fusion Energy, Department of Energy, Washington, D.C.

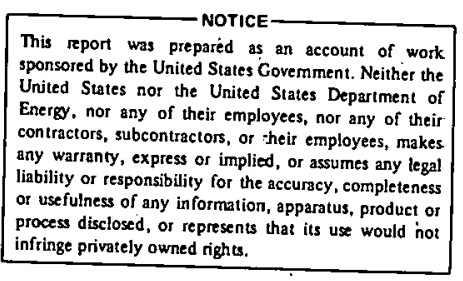

This report was prepared as an account of work unsored by the United States Government. Neither the contractors, subcontractors, of their employees, makes liability or respospress or implic or usefulness of any information, apparatus, product or infringe privately owned rights.
Date Published: August 1979

$$
\begin{gathered}
\text { Prepared by the } \\
\text { OAK RIDGE NATIONAL LABORATORY } \\
\text { Oak Ridge, Tennessee } 37830 \\
\text { operated by } \\
\text { UNION CARBIDE CORPORATION } \\
\text { for the } \\
\text { DEPARTMENT OF ENERGY }
\end{gathered}
$$


Printed in the United States of America Available frcm National Technical Information Service

U.S. Department of Commerce 5285 Port Rcyal Road, Springfield, Virginia 22161

Price: Printed Copy $\$ 6.50$.Microfiche $\$ 3.00$

This report was prepared as an accoun: of work sponsored by an agency of the United States Government. Neither the Uniied States Government nor any agency thereof, nor any of their employees, co ntractors, subcontractors, or their employees, rakes any warranty, express or implied, nor assumes any legal liability or responsibility for any thirc party's use or the results of such use of any information, apparatus, projuct or process disclosed in this report, nor represents that its use by such third perty would not infringe privately ownes rights. 


\section{CONTENTS}

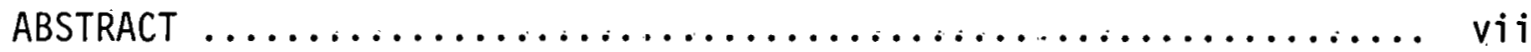

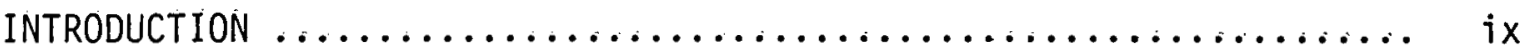

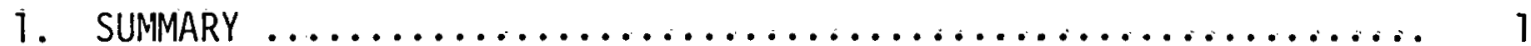

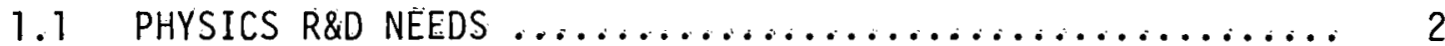

1.1.1 Plasma Confinement and Operation .............. 2

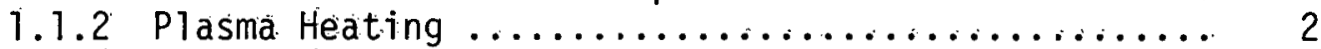

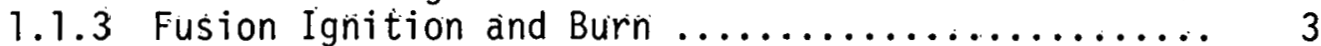

1.1 .4 Plasma Operation and Control ................ 3

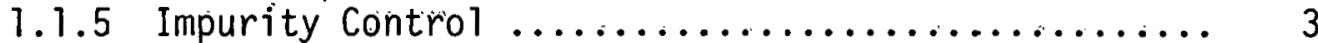

1.1 .6 Summary of Physics Needs .................. 4

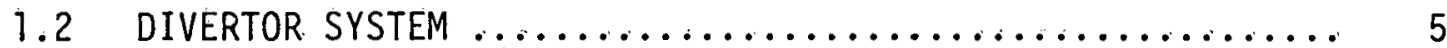

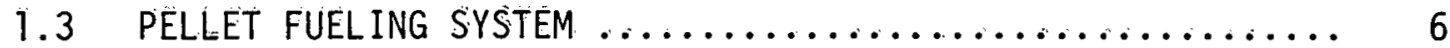

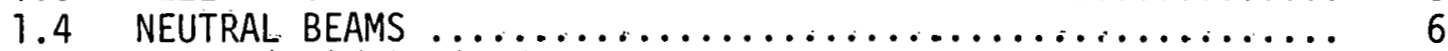

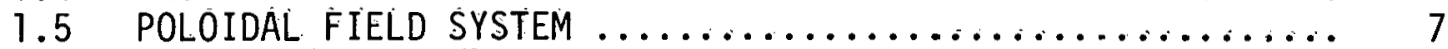

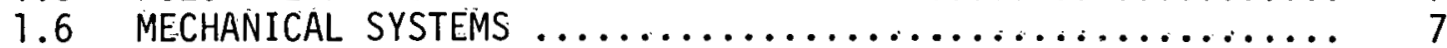

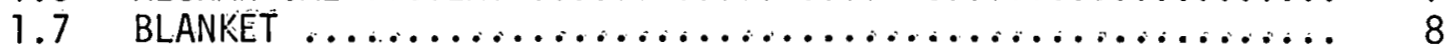

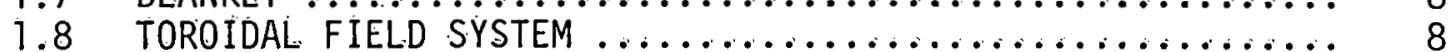

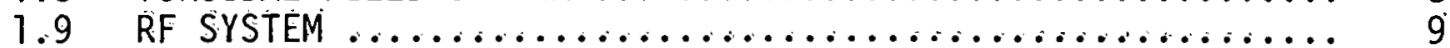

1.10 INSTRUMENTATION AND CONTROLS SYSTEM $\ldots \ldots \ldots \ldots \ldots \ldots \ldots . . \ldots$

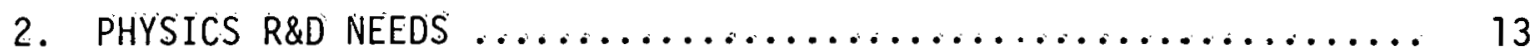

2.1 PLASMA CONFINEMENT AND OPTIMIZZATION $\ldots \ldots \ldots \ldots \ldots \ldots \ldots \ldots \ldots$

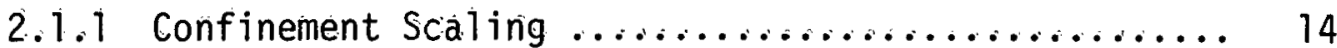

2.1.2 Stability Optimization ....................... 14

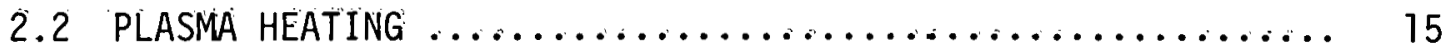

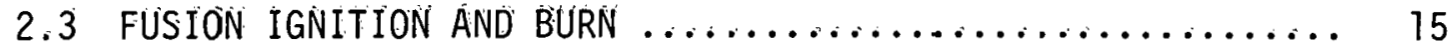

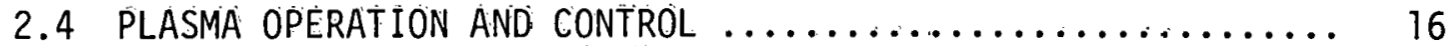

2.5 IMPURITY AND PARTICLE CONTROL $\ldots \ldots \ldots \ldots \ldots \ldots \ldots \ldots \ldots \ldots \ldots \ldots$

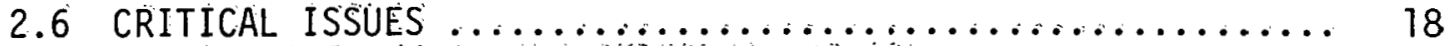

2.7 , ISSUES SPECIFIC TO THE REFERENCE DESIGN ............ 18

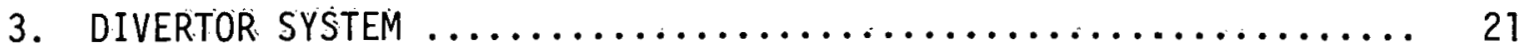

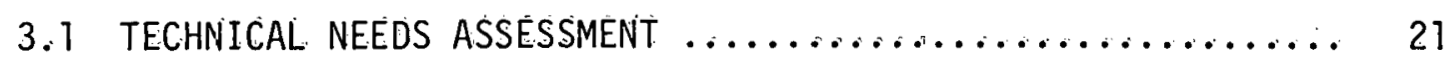

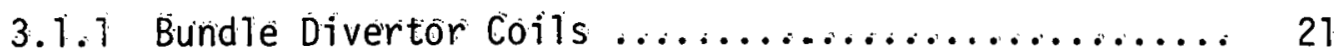

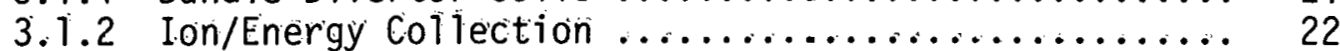

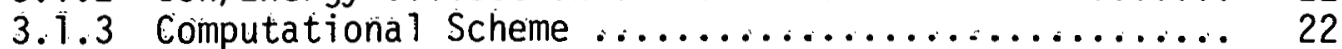

3.2 PROPOSED BUNDLE BIVERTOR SYSTEM $\ldots \ldots \ldots \ldots \ldots \ldots \ldots \ldots \ldots \ldots 22$

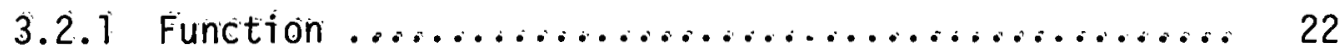

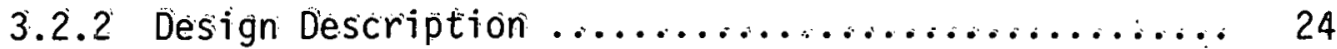

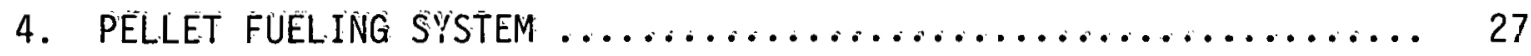

4.1 TECHNICAL NEEDS ASSESSMENT $\ldots \ldots \ldots \ldots \ldots \ldots \ldots \ldots \ldots \ldots \ldots \ldots$ 


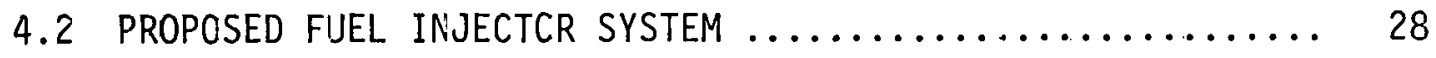

4.2.1 Function ................................. 28

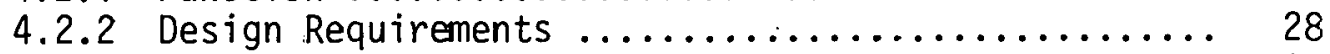

4.2.3 Design Description ......................... 29

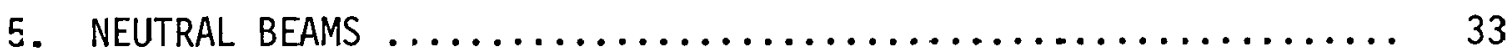

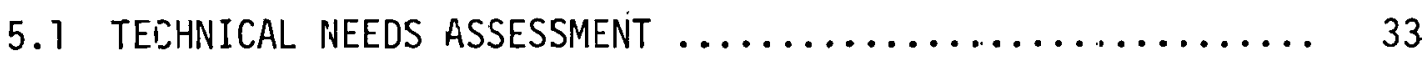

5.1 .1 Introduction ............................ 33

5.1.2 Status of System and Equipment Development and Availability .......................... 34

5.2 PROPOSED NEUTRAL BEAM HEATING SYSTEM $\ldots \ldots \ldots \ldots \ldots \ldots \ldots . . \ldots$

5.2 .1 Function ............................. 36

5.2 .2 Design Requirements $\ldots \ldots \ldots \ldots \ldots \ldots \ldots \ldots \ldots \ldots, 36$

5.2.3 Design Descripition $\ldots \ldots \ldots \ldots \ldots \ldots \ldots \ldots \ldots \ldots, 36$

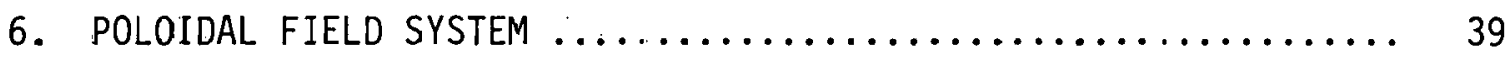

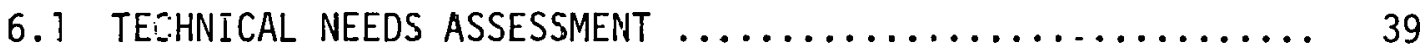

6.2 PRÓPOSED POLOIDAL FIELD SYSTEM $\ldots \ldots \ldots \ldots \ldots \ldots \ldots \ldots \ldots, 40$

6.2 .1 Function .............................. 40

6.2 .2 Design Fequirements $\ldots \ldots \ldots \ldots \ldots \ldots \ldots \ldots \ldots \ldots, 42$

6.2.3 Design Description $\ldots \ldots \ldots \ldots \ldots \ldots \ldots \ldots \ldots \ldots, 42$

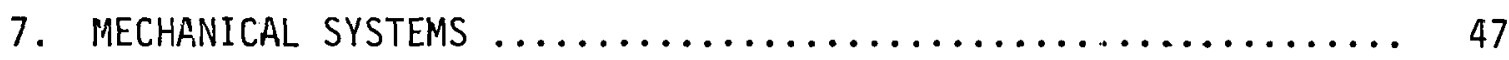

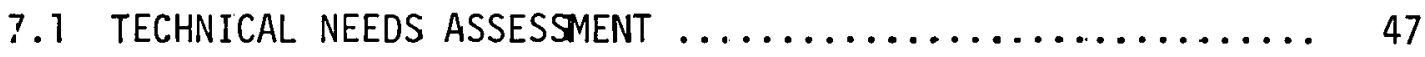

7.1 .1 Introduction ............................ 47

7.1 .2 Toroidal Vacuum Vessel $\ldots \ldots \ldots \ldots \ldots \ldots \ldots \ldots \ldots, 48$

7. 1.3 Machine Structure $\ldots \ldots \ldots \ldots \ldots \ldots \ldots \ldots \ldots \ldots \ldots, 49$

7.1 .4 Remote Servicing ......................... 50

7.1 .5 Shieiding ................................. 51

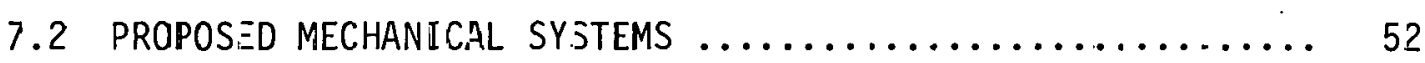

7.2.1 Limiter System ......................... 52

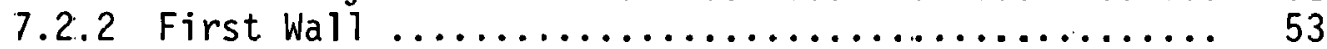

7.2 .3 Shield ........................................ 56

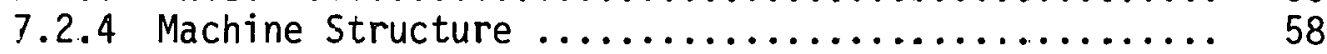

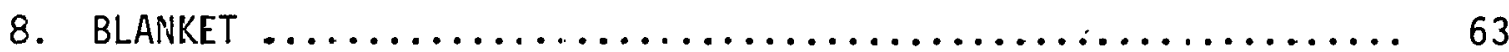

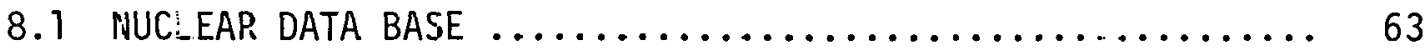

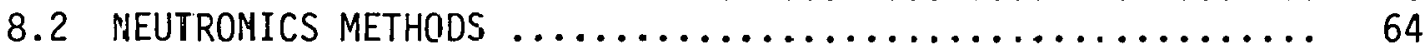

8.3 THERMAL-HYDRAULIC AND HEAT TRANSFER ANALYSIS $\ldots \ldots \ldots \ldots \ldots, 65$

8.4 PULSED MAGNETIC FIELD EFFECTS $\ldots \ldots \ldots \ldots \ldots \ldots \ldots \ldots \ldots, 65$

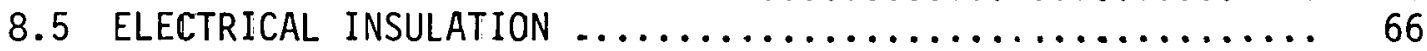

8.6 LIQUID LITHIUM CIRCULATION $\ldots \ldots \ldots \ldots \ldots \ldots \ldots \ldots \ldots \ldots \ldots \ldots \ldots \ldots \ldots, 66$

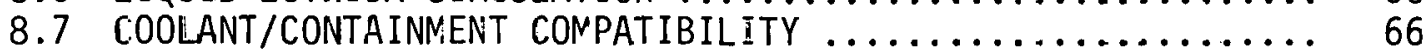

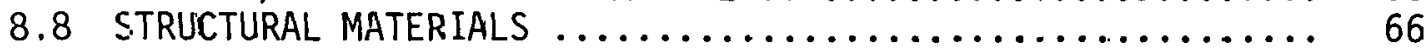

8.9 TRITIUM RECOVERY ................................... 67 


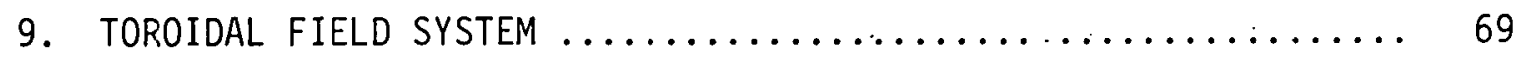

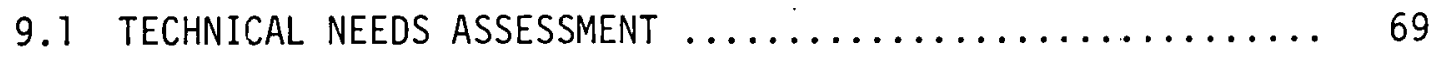

9.2 PROPOSED TOROIDAL FIELD SYSTEM $\ldots \ldots \ldots \ldots \ldots \ldots \ldots \ldots \ldots \ldots \ldots \ldots \ldots \ldots$

9.2.1 Function ............................. 70

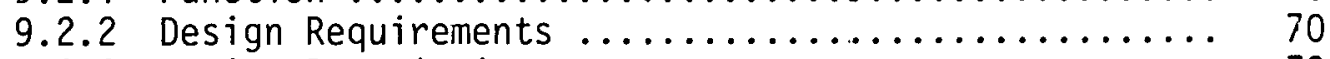

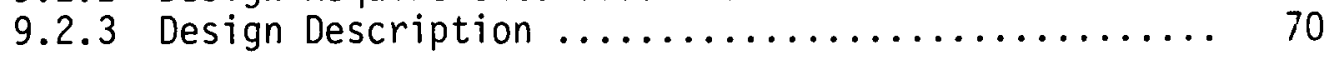

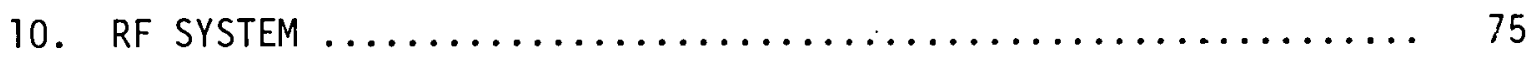

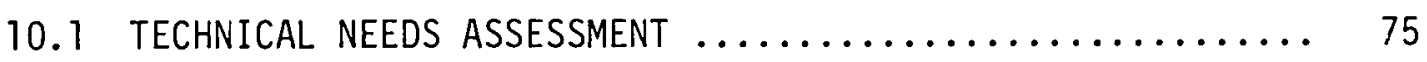

10.1.1 Quasi-Optical Oversize Wavequice ............ 75

10.1.2 Pressurization Window ..................... 77

10.1.3 Antenna Pattern ........................... 77

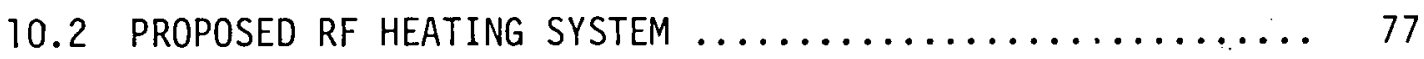

10.2.1 Function ............................ 77

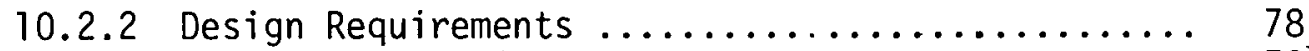

10.2.3 Design Description $\ldots \ldots \ldots \ldots \ldots \ldots \ldots \ldots \ldots .78^{-}$

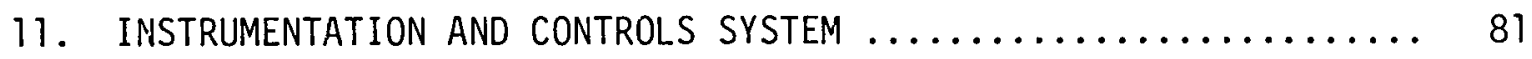

11.1 TECHNICAL NEEDS ASSESSMENT $\ldots \ldots \ldots \ldots \ldots \ldots \ldots \ldots \ldots . . . \ldots \ldots$

11.2 PROPOSED INSTRUMENTATION, CONTROLS, ANJ DATA

HANDLING SYSTEM $\ldots \ldots \ldots \ldots \ldots \ldots \ldots \ldots \ldots \ldots \ldots \ldots \ldots, 82$

11.2 .1 Function ............................. 82

11.2 .2 Design Requirements $\ldots \ldots \ldots \ldots \ldots \ldots \ldots \ldots \ldots, 8 \ldots \ldots,{ }_{82}$

11.2 .3 Design Description $\ldots \ldots \ldots \ldots \ldots \ldots \ldots \ldots \ldots .63$ 
THIS PAGE

WAS INTENTIONALLY

LEFT BLANK 


\section{ABSTRACT}

This document highlights the technical requirements of the key subsystems of the Reference Design for The Next Stap (TNS) and presents a preliminary assessment of the adequacy of the technical capabilities available to satisfy these requirements. Additioral information on the Reference Design and the FY 1978 TNS Program activities can be found in the associated technical memoranda, ORNL/TM-5720, ORNL/TM-6721, and ORNL/TM-6723 - ORNL/TM-6733. 
THIS PAGE

WAS INTENTIONALLY

LEFT BLANK 


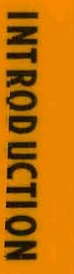




\section{INTRODUCTION}

The Next Step (TNS) represents that phase of fusion energy development in which the major emphasis would be directed toward engineering testing and demonstration. The objective of the TWS studies (initiated by the Department of Energy's Office of Fusion Energy) has been to define the characteristics and requirements of a facility dedicated to the engineering testing phase of fusion power development. For this reason, the TNS study results are providing a basis for defining an Engineering Test Facility (ETF). Because the scientific basis required for a TNS/ETF will first be available for the tokimak concept, the reactor core of the facility has been based on the tokamak concept. However, the commitment to an ETF with a reactor core based on the tokamak concept does not represent a commitment to tokamaks as the ultimate power reactor concept.

The TNS studies at Oak Ridge National Laboratory (ORNL) were initiated in FY 1977. During FY 1977 the Oak Ridye effort pursued scoping studies in three broad areas: plasma engineering, systems modeling, and program planning. Based upon the findings of the FY 1977 . efforts, it was judged that continued activities in the 0ak Ridge TNS program should be directed toward preconceptual cesign with particular emphasis placed on engineering feasibility. As a point of departure for the FY 1978 activities, a Baseline Design was sected, based on the systems modeling effort of FY 1977. The primary objective of our FY 1978 TNS effort has been to evolve the Baseline Design toward a preconceptual design. However, it is emphasized that the FY 1978 effort was not intended to lead to a completed preconcestual design. Therefore, the design resulting from this year's effort is referred to as a Reference Design, rather than as a preconceptual design.

This document highlights the requirements of the key subsystems of the TNS Reference Design and presents a preliminary assessment of the adequacy of the technical capabilities available to satisfy these requirements. Additional advancements in both physics and technology are necessary to establish the technical basis for the design of TNS. The major areas requiring special research and deveiopment (R\&D) emphasis are discussed for each subsystem. 


\section{SUMMARY}

The technical needs of the key systems of the Referer.ce Design are summarized. The goal of this continuing assessment effortl is the identification of potential barriers and technical gaps that may impede the successful implementation of the TNS program. This assessment employs both a rollback and rollforward determination of the needs for specific systems. The evaluation for each system is based on three questions:

(1) Wi11 the present and expected R\&D effort satisfy the requirements of the TNS program?

(2) Where are the major gaps in this R\&D area?

(3) How and when must these gaps be closed?

The level of effort directed toward the definition of the technical needs of the various systems was limited, and therefore a balance was made between depth and breadth of coverage for this year. Ten areas were addressed: physics needs, divertor system, pellet fueling system, poloidal field (PF) system, neutral beams, mechanical systems, blanket, radio frequency ( $r f$ ) system, toroidal field (TF) system, and instrumentation and controls system. The technical needs ard the R\&D advancements required in each area are summarized below.

It is noted that these assessments tend toward generic issues which will gain in specificity as the studies go forward. Two continuing themes appear in these assessments: (1) an increased emphasis on technology and engineering developments appears to be warranted to provide reliable, cost-effective components for TNS on a timely jasis, and (2) an expansion of the efforts to integrate the various physics results into a systems transient scenario and description is required to provide better guidance to R\&D needs. 


\subsection{PHYSICS R\&D NEEDS}

Recent advances in tokamak confinemert and heating experiments have provided optimism concerning the prospects for producing a controllable fusion core based on the takamak concept. Nevertheless, uncertainties still exist in essentially all of the physics assumptions adopted in the Reference Design, although these assumptions are considered rezsonable. This section briefly summarizes and discusies the phisics R\&D needs as suggested by the required inprovements in understanding. Highlighted are five physics areas in which even a rudimentary level of understanding is corsidered seriously lacking. These areas are ordered according to increasing level of uncertainty: plasma confinement, plasma heating, ignition and burn, operation and control, and impurity control.

\subsubsection{Plasma Confinement and Optimization}

This area deals with the plasma size, the TF strength, the plasma density, ard the plasma temperature required for ignition and burn in the Reference Design. These parameters are chosen in the context of their influence on plasma confinement. Substantial urderstanding in this area is already available, al though surprises in future experiments cannot be ruled out. Continued studies to improve tokamak confinement via improved stability by optimization of the plasma shape, profile, and beta are also being actively pursued. The evolution of high beta plasmas over long pulses is one of the topics on which work is still needed.

\section{1 .2 Plasma feating}

Heating with 150-keV, 50-MW deuterium teams for $6 \mathrm{sec}$ is assumed in the Reference Design. Recent neutral beam injection in the Princeton Large Torus (PLT), heating the plasma to near ignition temferatures, has increased confidence in these assumptions. Experiment $\bar{i}$ carroboration is needed for the theoretical calculations siowing that ignition can be reached with a neutral beam energy level of only $150 \mathrm{kel}$ using the low density or small radius heating scenario in the Reference Design. The 
physics of $\mathrm{rf}$ heating is presently less well understood, although vigorous experimental programs are in progress. The optimal combination of $r f$ and neutral beam heating also needs to be explored experimentally.

\section{i.1.3 Fusion Ignition and Burn}

Achieving $Q=1-2$ in the Tokamak Fusion Test Reactor (TFTR) will only provide a demonstration of the approach to ignition, while the Reference Design assumes ignition $(Q=5)$ and burn $(Q>5)$. An experimental understanding of the plasma physics of ignition and burn is needed. The study of the dependence of the burn process on the plasma parameters, the fusion alpha particle power deposition profile, the fueling rate, the deuterium-tritium (D-T) composition, and the method of fueling will provide the basis for burn control over long pulse times $\left(\sim 10^{2} \mathrm{sec}\right)$. Detailed numerical simulations, consistent with the proper confinement assumptions and the possible improvenents in TFTR achievements to $Q>2$, are needed to reduce the gap between present estimates and the actual demonstration of ignition and burn. An experimental test of ignition and burn still seems necessary to verify our assumptions.

\subsubsection{Plasma Operation and Control}

The desired plasma parameters, profiles, and cross sections through the phases of startup, heating, ignition, burn, and shutdown should be elucidated through studies in plasma confinement and optimization, heating, and ignition and burn. An understanding of the requirements of the plasma control along the desired path of oferation during the puise is presently lacking. Studies of methods to avoid fusion thermal runaway and plasma disruption and to reduce pulsed fields at the TF coils should be actively pursued.

\subsubsection{Impurity Control}

The plasma and impurity transport processes through the plasma edge/scrape-off region and in the divertors are presently the weakest area of physics understanding. The use of poloidal divertors will be 
studied in the Poloidal Divertor Experiment (PDX). However, the physics of the bundle divertors chosen for the Reference Design (because of their potential engineering advantages) and the impact of the divertors on plasma confinement must be studied at a level comparasle to that of PDY before a clear choice can be made for the TNS class of device. The imfurity production process involves interactions between the plasma and the wall/collector plates under large thermal power ioads. The long puise $\left(0,10-10^{2} \mathrm{sec}\right)$ behavior of these interactions and treir effects on the impurity control processes also need to be studied because presently successful methods using titaniun gettering may be linited to short pulses $(\sim] \mathrm{sec})$. Nondivertor impurity control schemes should also be expiored.

\subsubsection{Summary of Physics Needs}

On the basis of these discussions we can identify critical physics issles for the Reference Design. They include:

(1) Long pulse behavior of impurity production and transport under high thermal power in a diverted plasma.

(2) Long pulse evolution of high beta MHD equilibriz through substantia: flux diffusion, influenced by fusion alpha sarticle heating and various fueling sources.

We can also identify areas where some understanding can be developed via theoretical analyses and numerical simulations witt some confidence (altrough experimental studies in these areas would increase our confidence in the fusion plasma operation in the Reference Lesignj. They include:

(1) The physics of fusion ignition and burn (Q>5) for a time scale much :onger than the energy confinement tine.

(2) The physics of helium c.sh accumulation and transport in diverted plasmas. 
Finally, the physics/engineering studies during FY 1978 have suggested schemes that could have a favorable impazt on the reactor engineering and technology requirements. These studies point to the following desirable experimental verifications:

(1) Experiments to optimize the neutral beam injection process (such as the low density and small radius scenarios) that directly or indirectly corroborate the process of heating to ignition with beam energies of $2150 \mathrm{keV}$.

(2) Fueling experiments with partial pellet perietration.

(3) Experiments with a flexible PF coil system and superconducting TF coils to study ways to minimize the pulsed field during startup and disruption.

(4) Experiments with the use of plasma heating sources before and during current startup to increase the startup time and to reduce cost.

(5) Experiments with variable field perturbations (such as ripples in the toroidal field) as a method of controlling the runaway electrons and the plasma edge condition and improving access to the plasma core.

\subsection{DIVERTOR SYSTEM}

In the Reference Design, a bundle divertor was adopted for impurity control. The concept chosen for continuing study incorporates a liquid lithium jet system to absorb the large amount of diverted power and particles with a reasonable cross-sectional area. The assessment of the technical needs for the bundle divertor shows two areas of needed emphasis:

(1) Bundle divertor coil configurations that address

- the incorporation of larger coils that permit adequate scrape-off areas and allow room for adequate shielding,

- approaches to equalize the energy variation expected across the face of the lithium collection jets, and 
- methods of ceramic insulation of the coils that would ease the shieiding requirements.

(2) The liquid lithiun "shower" concept for ion/energy collection that addresses

- the expansion of the temperature range beyond the present $\sim 350^{\circ} \mathrm{C}$ limit where data are available on the collection of hydrcgen isotofe ions by liquid lithium,

- the ability of the "shower" to retain embedded alpha particles, and

- the ability of liquid lithium to pump neutral hydrogen isotopes.

\subsection{PELLET FUE_ING SYSTEM}

The fueling technique adop:ed in the Reference Design is injection of pellets of solid deuterium and tritium. Developments in this area haie providet encouraging results both in laboratory experiments and on the Impurity Study Experiment (ISX-A) tokamak. ${ }^{2}$ Con-inuing physics calculations and experimerits are needed to better de ine the injection requirements. The technical advancements required for ThS implementation are hightighted by three needed actions:

(1) Development of the potentially promising concepts of pellet injection by gas, mechanical, and electromagnetic methods of acceleration.

(2) Expansion of the data base on the physical properties of deuterium and tritiam pellets.

(3) Intensification of efforts to achieve equipment design innovations and simplifications that will lead to encanced equipment reliability, fabricability, and cost-effectiveness.

\subsection{NEUTRAL EEAMS}

The demonstrated success of neutral beam heating, in particular the recent PLT results, has provided a growing confidence that such systems can be used in future devices. However, the complexities of existing 
experimental beam lines raise issues concerning cost, reliability, and maintenance in the TNS context. The chief technical needs of neutral beam systems for the Reference Design are as follows:

(1) The capacity for $12.5 \mathrm{MW}$ per beam 1 ine for $5 \mathrm{sec}$ at energy levels up to $150 \mathrm{keV}$.

(2) Simple, more reliable systems that can obtain the necessary performance.

(3) Simple means of operating and controlling multiple beam lines.

(4) Design and fabrication concept $s$ that will minimize the difficulties of maintaining the beam lines in a reactor environment.

\subsection{POLOIDAL FIELD SYSTEM}

The PF system consists of seven sets of coils, each requiring separate power supplies. A combination of copper and superconducting coils (inside and outside the TF coil, respectively) is used; the interior copper coils permit a close coupling to the plasma. The key R\&D needs are:

(1) Pulsed superconducting coil technology to neet the Reference Design needs (e.g., $\dot{B} \sim \dot{2} \mathrm{~T} / \mathrm{sec}, B_{\max } \sim 8 \mathrm{~T}$ ).

(2) Simple electrical storage and supply circuitry for cost and reliability-improvements.

\subsection{MECHANICAL SYSTEMS}

The mechanical systems for the Reference Design include the machine structure, the secondary vacuum enclosure (SVE), the remote servicing equipment, and the shielding. The technology data base is largely available to meet the mechanical systems requirements of the Reference Design. However, the specific implementation of the design requirements requires significant expansion of data base, especially in two areas:

(1) Material properties in a high temperature, high neutron flux environment. 
(c) Remoze servicing equipment and methods for replacement and repa- $r$ of highly activated device components.

\subsection{BLANKET}

The Reference Design includes concepts for the first wall and bulk shield functions but does not address the requirements of a breeding bianket. The tlanket that must breed fuel, recover neat, and provide some shielding is being studiec in depth in the ORNL Tokamak Reactor Blanket Study, ${ }^{3}$ the objective of which is to develop a tilanket concept based on near-term technology that satisfies reliability and lifetime needs for a commercial reactor. A promising blanket concept nas been advanced anc preliminary aralyses support its potential for reactor application. This technical area is of particular vâlue to the engineering test phase of TNS, and a continuing blanket development effort is necessary. The blanket requirements in terms of surface and volumetric heat generation rates coup ed with the large number of power onioff cycles are challenging - even if the severe neutror irradiation effects are ignored. The following R\&D activities would be of particular benefit:

i1) Develapment of an experimental basis for promising blanket concepts via model testirg that includes some limited neutron environment. MHD effects can and should be tested for blankets emplojing liquid litrium.

(2) Cheracterization of the requirements imposed cn the blanket (i.e., particle loading and heat loads) in the event of an abnorral or disruption situation as well as far a normal cycle.

\subsection{TOROIDAL FIELD SYSTEM}

The Reference Design specifies an on-axis magnetic field of $5.3 \mathrm{~T}$, which is to be produced by i? superconducting TF coils with a rorizontal bore of $6.2 \mathrm{~m}$ and a vertical bore of $9.8 \mathrm{~m}$. This comination of number of coils and coil size yields a magnetic field ripple of $.5 \%$ at the plasma edge. The TF coils are assumed to be a pure tension: D-shape 
design with a trapezoidal cross section. The centering forces on the coils are reacted through a bucking cylinder. The major technology

needs associated with the TF system may be summarized as follows:

(1) Production of $\mathrm{Nb}_{3} \mathrm{Sn}$ conductor and manufacture of coils without strain degradation of properties.

(2) Designs for coil cooling that will avoid collections of helium bubbles and/or vapor in the liquid helium, which would result in degradation of the heat transfer.

(3) Means of coil protection, including the detection cf small normal zones, quench pressure in forced flow channel, or quench voltage in bath-cooled coils and the control of coil currents or fault forces.

\subsection{RF SYSTEM}

The use of $r f$ heating around $120 \mathrm{GHz}$ has been selected to aid plasma breakdown. Experimental substantiation of this assumption is critical and should be vigorousiy pursued because of the large potential cost benefits. Microwave power on the order of $1 \mathrm{MW}$ is suggested for deposition in the plasma region for $\sim 2 \mathrm{sec}$. Relatively efficient waveguides and flush-mounted arrays can be designed to accomplish the power transmission. The major R\&D areas that shculd be pursued include:

(1) Development of power tubes to achieve $200 \mathrm{~kW} \mathrm{cw}$ per tube at $120 \mathrm{GHz}$.

(2) Development and component testing of the elements required to transfer the rf power from the sources to the plasma, including: oversize waveguides, modal filters, materials that can tolerate the reactor environment, pressurization windows, and antennas.

\subsection{INSTRUMENTATION AND CONTROLS SYSTEM}

This system is required to automatically control the device through all its phases of operation. Instrumentation is provided for controlling functions and for diagnostics. The data from tre instrumentation are 
processec and either displayed or stored for later en-call display. The philosopry cf operation is that of semiautonomous subsystem controls and diagrostics operating under the supervision of a central computerized control. A significant emphasis in the development of this system must be the necessary designs for fail-safe operations and degraded mode options.

Table 1.1 is a list of major parameters thay may need to be measured, along with some sensing concepts that should be studied for use in the reactor environment.

Table 1.1. Parameters to be measured and candidate sensing concepts

\begin{tabular}{ll}
\hline \multicolumn{1}{c}{ Parameters } & Candidate sensing cancepts \\
\hline $\begin{array}{c}\text { Plasma density as a function } \\
\text { of time and plasma radius }\end{array}$ & $\begin{array}{c}\text { Thomson sca-tering, } \\
\text { microwave abscirption }\end{array}$ \\
Plasma location & Inductive pickuf loops \\
Average plësma current & Rogowski coiis \\
Plasma icn anc electron & Thomson scattering, \\
temperatıres as functions & soft x-ray detectors \\
of time and plasma radius & Inductive pickup loops \\
Magnetic field strengths & Activation fals, collimating \\
Neutron flux at first wall & tube and detectars \\
\hline
\end{tabular}

Future investigations must include more study of the problems of disruptions, aicorts, and shutdown ard of the difficulties of defining sensors and instrumentation systems that can survive the reactor environment and meet the accuracy and response demands of the controls and diagnostics. Thus, the primary R\&D needs include:

(1) Development of a hybrid computer instrumentatior and controls (I8C) simulation, benchmarked with experiments and expansion to long pulse calculations and experiments.

(2) [ievelopment of instrumentation that can operate in the expected reactor environment. 


\section{REFERENCES}

1. Oak Ridge TNS Program Staff, Draft Program Plar. for TNS - The Next Step Ajter the Tokamak Fusion Test Reactor. Pcrt II - R\&D Needs Assessment, ORNL/TM-5983, Oak Ridge, Tennessee (December 1977).

2. S. L. Milora et a1., Hydrogen Pellet Fueling Experiment on the ISX-A Tokamak, ORNL/TM-6496, Oak Ridge, Tennessee (October 1978).

3. P. B. Mohr et al., Tokamak Blanket Design Studı: FY 78 Summary Report, ORNL/TM-6847 (1979). 
THIS PAGE

WAS INTENTIONALLY

LEFT BLANK 
厗 


\section{PHYSICS R\&D NEEDS}

This section discusses the R\&D needs in fusion plasma physics to ensure successful plasma operations in a device like the TAS Reference Design. This discussion points to the additional knowledge and understanding required to reduce the uncertainties in our physics assumptions, extrapolations, and theoretical calculations to a level acceptable for economy and risk prevention. The needs discussed here are not.compared with specific present and planned physics experiments in the magnetic fusion community. However, some preliminary evaluation is included to highlight those physics areas where serious gaps are considered most likely to exist.

Recent advances in tokamak plasma confinement and heating experiments have resulted in reactor level temperatures and densities in separate devices. These have provided optimism concernins the prospects for producing a controllable fusion core based on the tokemak concept. It should be emphasized that uncertainties still exist in essentially all of the physics assumptions adopted in the Reference Design. Our discussion is concerned with the presently conceived Reference Design and approaches to plasma operation. However, uncertainties of a more general kind also exist; because our experiences in tokamak operations are limited to physics oriented experimental devices, it should not be surprising that an engineering oriented reactor device may dictate approaches drastically different from our present conceptions.

For convenience, the physics issues are divided into five categories, ordered according to increasing levels of uncertainty: plasma confinement and optimization, plasma heating, fusion ignition and burn, dynamic scenarios of operation and control, and impurity and particle control.

\subsection{PLASMA CONFINEMENT AND OPTIMIZATION}

This area deals with the achievable plasma zemperature, density, and confinement times, and provides estimates of the plasma size, TF strength, and heating power required for ignition and burn. Two areas are of interest here: confinement scaling and stability optimization. 


\subsubsection{Con:inement Scalirig}

Recent tokamak confinement experiments have indicated that the ion heat corduction is nearly neoclassical, while the electron heat conduction seems to scale empirically like $n^{-1} T^{-\alpha}$, where a apparently ranges from 0 to 1 . Particle diffusion seems to behave similarly to the enpirical e.ectron scaling. Without an understanding of the processes leading to the empirical scaling laws, the extrapolation of these laws tc the TVS ieference Design would involve a significant level of risk. The unce-tainties, although they may not be critical, may result in a wide envelope of device parameters. Continued experimental and theoretical studies of the tokamak confinement processes are called for to ensure an economical design with acceptable risks.

\subsubsection{Stability Optimization}

The concerns about the effects of trapped particle instabilities have decreased because of recent results on PLT that indicate nearly neaclassical ion energy confinement in reactor level collisionality regimes. MHO instabilities due to the presence of minute plasma resistivity have been observed in mariy tokamak experiments. They are seen to have sensitive cependences on the profiles of current and safety factor. At high levels of instability, they lead to degradation of plasma confinement and eventually to sudden disruptions of the plasma current. While sigrificant progress has been made in understanding the properties and consecuences of resistive MHD instabilities, the scaling to reactor level plasmas is by no means understood.

It is generally assumed that the pressure driven, ideal MHD instabilities will place a stringent limit on the attainable plasma beta. The magnitude of beta has a dramatic impact on the size, the field strength, and hence the cost of a reactor. Understanding the dependence of the beta 1 imit on parameters such as crass section shape, plasma profiles, and aspect ratio is of great importance to the Reference Design. The assimed value of $B=7 \%$ is consistent with the latest calculations and is presently considered adequate for marcinal reactor economy. A significant increase in the beta limit is highly desirable. 
The designed pulse length of $500 \mathrm{sec}$ is expected to be comparable to the plasma resistive time scale. The evolutior of high beta plasmas over this time scale is one of the topics on which work is still needed.

\subsection{PLASMA HEATING}

Neutral beam heating with 50 MW of 150-keV deuterium beams for $6 \mathrm{sec}$ is calculated to be adequate to ignite a clean plasma in the Reference Design. The recent success of the PLT experiments in reaching near ignition ion temperatures has increased the confidence in this assumption. However, the success of using 150-keV beams depends on the success of the heating scenarios proposed: expanding radius heating or low density heating. Early experimental verification of these heating scenarios is desirable to determine whether higher energy beams are needed.

In comparison to neutral beam heating, the physics of $r f$ heating is presently less well understood but has the potential for more economical and convenient applications to a reactor grade tckamak; rf heating may also be used to supplement neutral beam heating for increased reliability and reduced cost. The optimal combination of rf heating and neutral beam heating needs to be explored experimentally.

\subsection{FUSION IGNITION AND BURN}

Achieving $Q=1-2$ in TFTR will provide a demonstration of the approach to ignition when the fusion alpha particles become the dominant heating source near the center of the plasma. The information obtained, while extremely valuable for understanding the ignition process, may rot be adequate for reducing the uncertainties in extrapolating to ignition $(Q \sim 5)$ and burn $(Q \rightarrow \infty)$ to an acceptable level. The process of ignition and burn may depend strongly on the electron and ion temperatures, the plasma density, the fusion alpha particle power deposition process, and the D-T composition. Experimental study of near ignition processes should not only help confirm the confinement scaling laws and the heating scenarios, but also provide information on optiral regions of plasma parameters for ignition. 
A difficult question has to do with the level of naar ignition to be achieved before the obtained information can be consiciered adquate for extrapolation to ignition and burn. If demonstration of full ignition is not required for this, it may become desirable to improve the performance of TFTR and the Joint European Torus (JET) to approach $Q \sim 5$ for as long a pulse as possible. Detailed numerical modeling and simulation of the experimental results are needed to bridge the gap between present estimates and the actual demonstration of ignition and burn.

\subsection{PLASMA OPERATION AND CONTROL}

It mat be overly optimistic to expect that an a fequate understanding of the three categories discussed above will be obtained in the early 198Js. However, it is possible that plassma operation tr.rough the phases of startup, heating. ignition, burn, and shutjown in a reactor grade tokamak will be drastically different from our present conceptions, which are based on physics oriented devices. Desirable ciperating scenarios may be discovered through a concerted study of the reactor engineerirg, technology, and physics concepts, but the scenarios thus proposed must be experimentally verified before they are applied to reactcr grade plasmas.

Closely coupled to the plasma operation are the plasma control requirements $\equiv$ long the desired path in the parameter space during the pulse. Critical areas include fusion thermal runaway, plasma oisruption, and the pulsed fields at the TF coils. Lack of clear definition of the requiremenzs in these areas may fresent overly stringent requirements on engineering and technology, making a reactor grade tokamak unacceptably costly and unreliable.

\subsection{IMPURITY AND PARTICLE CONTROL}

This is presently the weakest area of physics understanding. The area deals with impurity production at the plasma-wall interface and with impurity transport through the plasma edge/scrape-off regicn and in the divertor chamber. Planned experiments will study various impurity 
control methods both with and without divertors. Significant improvements in our understanding of this area are expected. However, these experiments are limited to short pulse times ( $\sim \mathrm{sec}$ ) at relevant power levels $\left(2100 \mathrm{~W} / \mathrm{cm}^{2}\right)$ and are not likely to provide adequate information for a design to operate in a clean, long pulse, high beta, D-T fusion plasma, as assumed in the Reference Design.

Bundle divertors have been chosen for the Reference Design because of their potential engineering advantages, the desire to scope the requirements of bundle divertor technology, and tre lack of quantifiable estimates of impurity control requirements: This has led to the design assumption in the TNS study that the bundle divertors offer sufficient flexibility to permit the evolution of a workable reactor grade impurity control method. The bundle divertor may extract a relatively small flux bundle from the plasma edge. This can be an attractive feature in the event that only fractional efficiency in particle unloading, impurity screening, and heat unloading is required for reactor impurity control and ash removal. However, the bundle divertor may introduce field ripples that degrade the confinement properties of the toxamak.

Nondivertor impurity control approaches shouid also je pursued actively because of their intrinsic simplicity. However, these approaches are likely to be inflexible and may not apply to long pulse operations because of helium and impurity buildup. It may be premature to rule out the possible usefulness of impurity control witho'st divertors, but we cannot rule out the need for some divertor oriented impurity control method for long pulse, reactor grade plasmas. Research and development of divertor physics and technology should be pursed actively to provice the data base needed for proper reactor design and operation of impurity control schemes.

A closely related topic of interest is fueling. Recent pellet fueling experiments on ISX-A have demonstrated the feasitility of this technique, al though vigorous efforts are needed before reactor application can become a reality. Combined with gas puffing, pellet injection may offer the needed flexibility. in particle control in diverted reactor plasmas. 


\subsection{CRITICAL ISSUES}

On the basis of these discussions we can identify zritical physics issues for the Reference Design. They include:

(1) Long puise behavior of impurity production and transpert under high thermal power in diverted and nondiverted plasmas.

(2) Long pulse evolution of high beta MHD equilibria through substantial flux diffusion and influenced by fus"on alpha particle heating and variaus fueling sources.

We can also identify; areas where some understanding can be developed via theoretical analyses and numerical simulations of low $Q$ experimental results. However, experimental studies in these areas would increase our corfidence in the fusion plasina operation in the Reference Design. They include:

(1) The physics of fusion ignition and burn $(Q>5)$ for a time scale much longer than the energy confinement time.

i2) The physics of heli $\mathrm{mm}$ asi accumulation and transport in diverted plasma.

\subsection{ISSUES SPECIFIC TO THE REFERENCE DESIGN}

Finally, the TNS physics/engineering studies during FY 1578 have suggested schemes that could favorably imfact the reactor engineering and technology requirements. These studies point to the following desirable experimental verifications:

(1) Experimerts to optimize the neutral beam injection process (such as the low density and small radius scenaricis) that directiy or indirectly corroborate the process of heating to ignition with beam energies of $\sim 750 \mathrm{keV}$.

(2) Fueling experiments with partial pellet penetration.

(3) Exferiments with a versatile PF coil system and superconducting TF coils to study ways to minimize the pulsed field during startup and disruption. 
(4) Experiments with the use of plasma heating sources before and during current startup to increase the startup time and to reduce costs.

(5) Experiments with variable field perturbations (such as ripple in the toroidal field) as a method of controlling the runaway electrons and the plasma edge condition and of improving access to the plasma core. 
THIS PAGE

WAS INTENTIONALLY

LEFT BLANK 


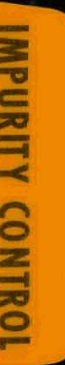




\section{DIVERTOR SYSTEM}

\subsection{TECHNICAL NEEDS ASSESSMENT}

Study in this area has concentrated on the bundle divertor option. (The poloidal divertor approach was studied in some deptr last year.) Bundle divertors have been emphasized primarily because they remove the ion and energy collection functions to a region outside the space bounded by the TF coils.

In the consideration of previous bundle divertor concepts, a review of the data on solid ion absorbers (titanium and zirconium) suggests that the use of such absorbers in reactors is unlikely because of (1) the anticipated degradation of thermal and mechanical properties with cyclic saturation and regeneration and (2) the excessive times and temperatures to regenerate these absorbers. The bundle divertor concept proposed for the Reference Design is a system involving liquic lithium droplets traveling through the volume of interest, which can obviate these problems. The proposed concept is described in Sect. 3.2.

The technical needs that must be addressed for TNS cre summarized below.

\subsubsection{Bundle Divertor Coils}

The use of larger coils is desirable, but magnetic field perturbations near the central region of the plasma must be minimized. Studies of configurations to fulfill these conditions are needed.

The plasma guided by the diverted flux bundle is expected to produce sharp peaking of surface heat loads. Metiods must be found to reduce this peaking in the target region as well as to expand the field generalily.

Ceramic insulation must be developed for water-cooled copper coils, based on studies showing that the shielding for superconducting coils and/or the organic insulation for regular coils tend to close up the ion flow passage through the bundle divertor coils. 


\subsubsection{Ion/Energy Collection}

The present limit on the temperature range where data are available on the collection of hydrogen isotope ions by liquid 1 ithidm is $\sim 350^{\circ} \mathrm{C}$. This must be extended to higher lithium temperatures.

Tre ability of the prozosed concept to retain embedded alpha particles must be addressed. This will involve defining the concept for pumping the collezted droplets and measuring the collection and removal of ions. Alsa, the ability of the liquid lithium to pump neutral hydrogen isotopes must be ascertained.

An approach that uses cold walls to retard backstreaming of 1 ithium into the plasma region has been suggested; the merits of this approach must be determined.

Experiments should be conducted on the passage of draplets through nonLni iorm magnetic fields.

Finally, the data on solid absorbers should be extenced for possible use in TNS: where full reactor requirements would not be imposed.

\subsubsection{Computational Schere:}

A computaticnal model should be developed to optimize divertor coi's so that perturbations of the magnetic fields can be minimized while the necessary nullirg and reversal of the toroidal magnetic field are accomplished. This might be an interactive scheme involving cathode ray tube (CRT) display or nathematical minimization or masimization schemes.

\subsection{PROPOJED BUNDLE DIVERTOR SYSTEM}

\subsubsection{Funstion}

The divertor system (1) minimizes the interaction between plasma particles and the walls oi the plasma containment vessel and ( $\bar{c}$ ) diverts and collects impurities emanating from the walls. As a corrsequence, it is necessary to collect the diverted particles and to handle the heat load associated with these diverted particles. For design purposes, it 
has been assumed that the upper limit on the heat load to the divertor is equivalent to the alpha particle energy. The actual heat load to the divertor is estimated to be $50 \%$ of the alpha particle energy, with the remaining 50\% absorbed by the first wal1. The divertor system parameters are listed in Table 3.1.

Table 3.1. Divertor system parameters

Geometric

Number of bundle divertors

1 operating, 1 or 2 on standby or being cleared

Divertor opening of deposited lithium

Shield thickness $0.4 \mathrm{~m}$

Flux bundle expansion ratio

$0.4 \mathrm{~m}$

90

Performance

Ripple at plasma centerline (due to divertor)

$3 \%$

Plasma edge particle loss rate (energy transport)

$3 \times 10^{23}$ particles $/ \mathrm{sec}$

Plasma fraction recycled at edge as neutrals

$85 \%$

Maximum heat load (design value)

226 MW

Actual heat load

$113 \mathrm{WW}$

Electromagnetic

Coil type

Ampere-turns

Water-cooled copper

$12 \times 10^{6}$

Thermal

Heat flux to divertor collector

$10 \mathrm{MN} / \mathrm{m}^{2}$

Divertor collector surface area

$22.6 \mathrm{~m}^{2}$

Lithium temperature rise

$114^{\circ} \mathrm{z}$

Divertor coil coolant

Water

Divertor coil ohmic heating

$70 \mathrm{MN}$

Shield coolant

Water

Particles pumped by divertor

$4.6 \times 10^{22}$ particles $/ \mathrm{sec}$ 


\subsubsection{Design Description}

The bundle divertor shown schematically in Fig. 3.1 is the type crosen for the Reference Design. It is composed of two coils, side by side, with oppasite current flows that provide a strong short-range majnetic field, which is superimposed on the toroids l field in the pliasna to produce zero field close to the external surface of the plasna. The resulting field distortion allows a burdle of field lines to be extracted and form a loop external to the plasma clong which cr.arged particles trappec on the lines can travel.

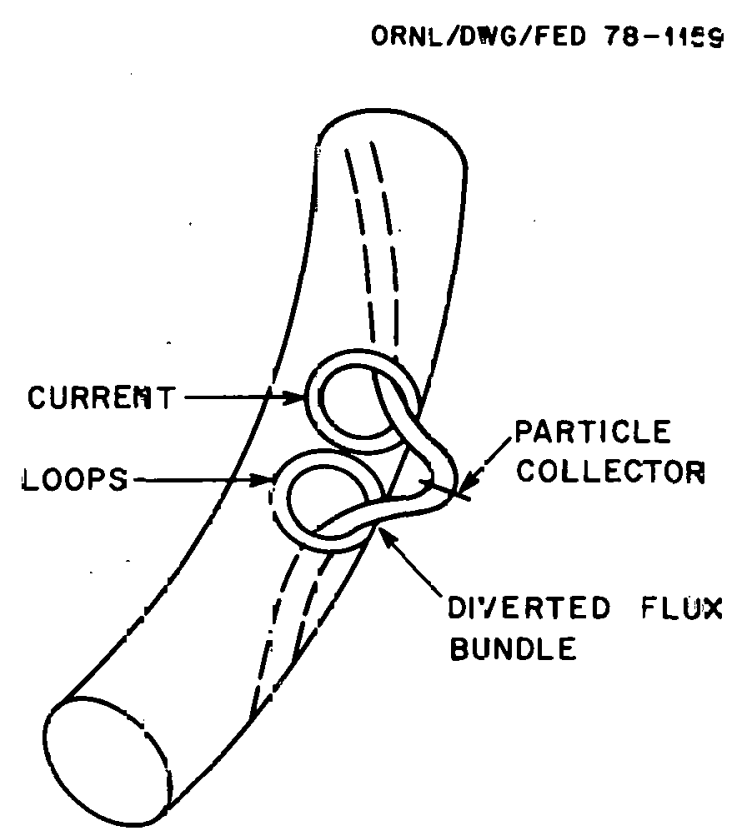

\section{BUNDLE DIVERTOR}

Fig. 3.1. Bundle divertor.

In a reactor envircnment, the divertor will be subjected to a high level of radiation, and some shielding ( $\mathrm{cn}$ the order of $1 \mathrm{~m}$ for superconducting coils) will te required to pratect conductors and insulation from cumulative damage. This shielding requirement leads to oversize divertor coils, which create excessive perturbation of the field lines in the plasma. However, divertor coils of the proper size cannot be adequately shielded. A shield thickness of $0.4 \mathrm{~m}$ appears to be a 
feasible compromise. This thickness precludes the use of superconducting and normal cryogenic coils but permits the use of water-cooled copper with a shortened life.

The divertor will expand the diverted magnetic field to reduce particle and energy flux. For the purpose of design, it is assumed that the divertor must absorb all the power associated with the alpha particles (226 MW). This power is assumed to leave the plasme through a particle loss rate at the plasma edge of $3 \times 10^{23}$ particles/sec. It is further assumed that $85 \%$ of these particles recycle as neutral gas. The number of plasma particles that the divertor is required to pump is therefore $4.6 \times 10^{22}$ particles/sec.

For a solid absorber, the getter material must either constitute or be a part of a heat exchanger, which requires material having good strength and thermal conductivity, since it will be subjected to at least $10^{5}$ cycles of absorption and desorption. Corsideration of data on hydrogen embrittlement of structural materials (for one absorption cycle) suggests that this will seriously degrade the material. Also, the times and temperatures for regeneration of getters $\left(700-1000^{\circ} \mathrm{C}\right.$ for several hours) tend to indicate infeasibility. Therefore, these functions will be accomplished by an array of lithium jets, shown schematically in Fig. 3.2. For the absorption of a heat flux of $10 \mathrm{MW} / \mathrm{m}^{2}$, there must be $22.6 \mathrm{~m}^{2}$ on which the expanded, diverted magnetic field impinges. The expansion requirement leads to a system that has much in common with the direct conversion approaches proposed for advanced fuel concepts. 


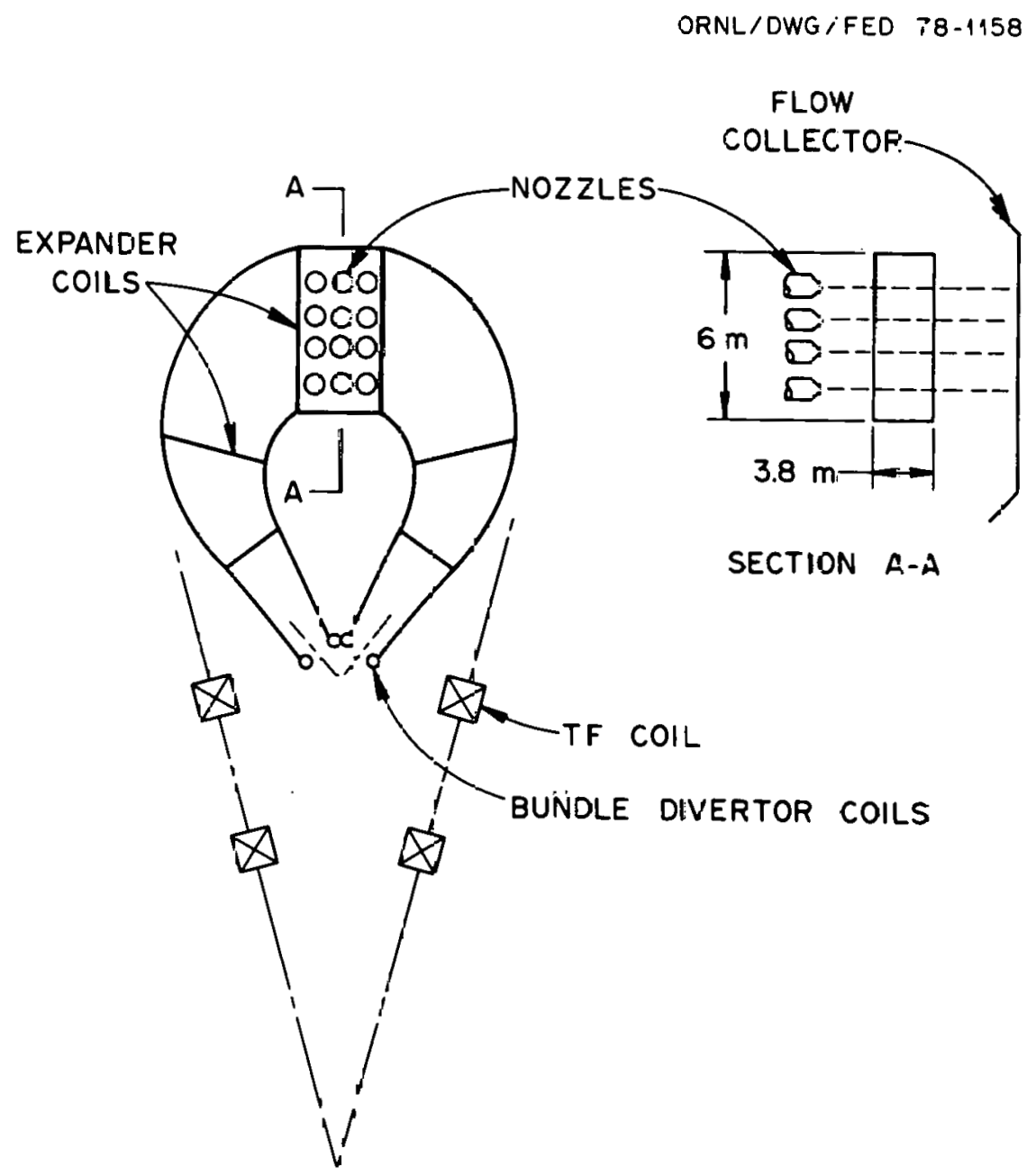

Fig. 3.2. Lithium jet energy collection system for bundle diverior. 
总 


\section{PELLET FUELING SYSTEM}

\subsection{TECHNICAL NEEDS ASSESSMENT}

Pellet injection has been chosen as the fuelirg technique for the TNS Reference Design. The requirements for pellet injection systems result from the plasma characteristics and their control o\%er the penetration depth, along with the attendant partic e spatial and temporal deposition. Studies and R\&D needs in these areas are discussed in Sect. 2 .

Specific requirements for the pellet composition, velocity, and injection rate will be established by the burn scenario. Thus, a primary area of concern for the pellet injector system is the pellet characterization, which includes pellet formation, size, composition, and thermal and mechanical properties. Early definition of these requirements is important so that their impact on injector design can be taken into account and the ability of the candidate ideas to meet these needs can be evaluated.

The pellet injector concepts that now offer the most promise of meeting the needs of a fusion reactor are the pneumatic gun, the pressurized fluid injector, and the centrifugal slinger. It seems desirable to investigate all three concepts in increasing depth, since none has been advanced much beyond laboratory studies and tests.

The pneumatic gun concept was used in a short feasibility test on ISX-A in which pellets were injected into the plasma with encouraging results. Experiments are planned on ISX-B to assess the injection performance more fully. The recent study and development test results strengthen the consideration of the pneumatic gun as a candidate injection system. The fluid injector ideas need a different perspective in some respects, because the pellet formation is a more integral part of the actual injection technique. The centrifugal slinger approach has been selected for the TNS Reference Design, but it should be noted that the recommended R\&D effort includes consideration of the other leading candidates as well, because all developments are in such an early stage that firm choices cannot be made with confidence. 
These concepts have Deen studied in light of recent results from fueling calculations that have increased confidence about finding sienarios that require peliet velocities of $\leqslant 1000 \mathrm{~m} / \mathrm{sec}$. All three concepts show promise of being able to meet these velocity requirements.

In addition to the physics issues discussed in Sect. 2, the technical needs in pellet iniection for TNS are highlighted by three areas of recommended action:

(1) Evaluation, by calculation and experiment, of the potential of aiternate concepts of pellet injection, such as gas and mechenical methods, to provide the frozen D-T pellets at a controlled velocity and rate of delivery to the plasma.

(2) Expansion of the data base on pellet formatian and handling and on mechanical and thermal characteristics under injection conditions.

13) Intensification of efforts to achieve equipment design innovations and simplifications that will lead to enhanced equipment reliability, fabricability, and cost effectiveness.

\section{2 ?ROPISED FUEL INJECTOR: SYSTEM}

\subsubsection{Function}

The fuel injector injects D-T fuel in the form of frozen sellets to reflenish any fuel lost or consumed during a burn, trus maintaining the plasma characteristics consistent with the fusion pracess. The pellets must be properly sized and Jelivered with sufficient velocity =o penetrate the hot plasma to an appropriate depth. The fuel injector parameters are given in Table 4.1.

\subsubsection{Design Requirements}

The injestor must be shielded from electrical and magnetic fields in a manner triat provides unimpaired operation during steady state or in transient environments. The unit electrical circuits must be protected from fault or induced electrical overloads by adequate fuses or circuit breakers. 
Table 4.1. Fuel injector perameters

\begin{tabular}{ll}
\hline Steady-state burn & $500 \mathrm{sec}$ \\
Cycle time & $560 \mathrm{sec}$ \\
Fueling rate (during burn) & $0.185 \mathrm{~g}$ ' $\mathrm{sec}$ \\
Pellet shape & Spherical \\
Pellet diameter & $\sim 0.5 \mathrm{~cm}$ \\
Pellet velocity & $1000-2000 \mathrm{~m} / \mathrm{sec}$ \\
Repetition rate & $16 \mathrm{pellets} / \mathrm{sec}$. \\
\hline
\end{tabular}

Operating temperatures of units of the injector must be controlled by passive or active thermal control systems. The recommended cooling media are liquid nitrogen ( $\left.\mathrm{LN}_{2}\right)$ and liquid helium (LHe).

The injector must have adequate shielding to limit neutron activation of components. Modular designs for components or parts subject to radioactivity or tritium products will provide for ease in maintenance.

There must be no particles from moving mechanical assemblies, lubricants, or outgassing products that could migrate to the vacuum vessel. Vacuum pumps maintain the injector enclosure at a pressure equal to or lower than that of the vacuum chamber and exhaust evaporated $D-T$ products and gaseous products.

\subsubsection{Design Description}

The fueling method chosen for the Reference Jesign is the injection of pellets of solid deuterium and tritium. Recent theoretical studies indicate that pellet penetration may be reduced to about 0.2 times the plasma radius, compared to earlier estimates requiring penetration to the plasma centerline. This may result in reductions of pellet velocity to as low as $1000 \mathrm{~m} / \mathrm{sec}$.

The fuel pellet injector mechanism provides the desired injection velocity by exerting centrifugal force on a fuel pellet. A rapidly rntating disc spinning on a vertical axis captures the fuel pellet, which is introduced to the upper face of the disc close to the central hub. The pellet is accelerated along a formed track mounted on the 
disc"s upper face by centrifugal force and leaves the disc through a tube connected to the plasma chamber. The rotational speed oi the disc, the disc diameter, the track geometry, and the pellet characteristics govern the ejection velocity and irajectory. Princifal subassemblies of the injector mechanism are the notating disc with its drive, the device that forms the solid (frozen) pel'ets from deuterium and trit'um gases, anf the pellet cutter/deliverer that propels the D-T pellet to the imseller.

The entire assembly is housed in a vacuum-tight container. A vacuum-tight tube connects the main unit to the plasna coember and serves as a clear passage =or pellet entrance to the plasma chamber. While the pellets are being injected, the pellet injection device is subjected to direct therma? radition and neutron streamirig. The entire vacuun-tight housing and the interfacing subsystems are shielced from possible radioactivity in the vicinity of the device for personnel protection.

The pellet injector requires these supporting components and subsystems: vacuur pumping, liquid helium supply and plumbing, electrical power, instrumeritation arid controls, basic structure to support injector housing and passive shielding, and filtration and recycling for handling misfired pellets or gaseaus tritium products. Figure 4.1 illustrates the funct: ins of the system.

The 'njecter and major subsystem components wil' be assembled as a single renovable unit, with provisions for remote renoval and capping of subsystem lines when the pellet assembly is removed for service. The injector will be designed in functional modules that iacilitate disassenbly and repair. The housing in which moving parts are enclosed must coritain any component parts that fail and must protect tritium system and lines fron damage or lass of tritium from the injectcr system.

iwo ar more pellet injector systems will be installed with one unit always in stándby mode. Suitable instrumentation anc controls will warn of malfunction in the operating unit and switch the standby unit into operation. 


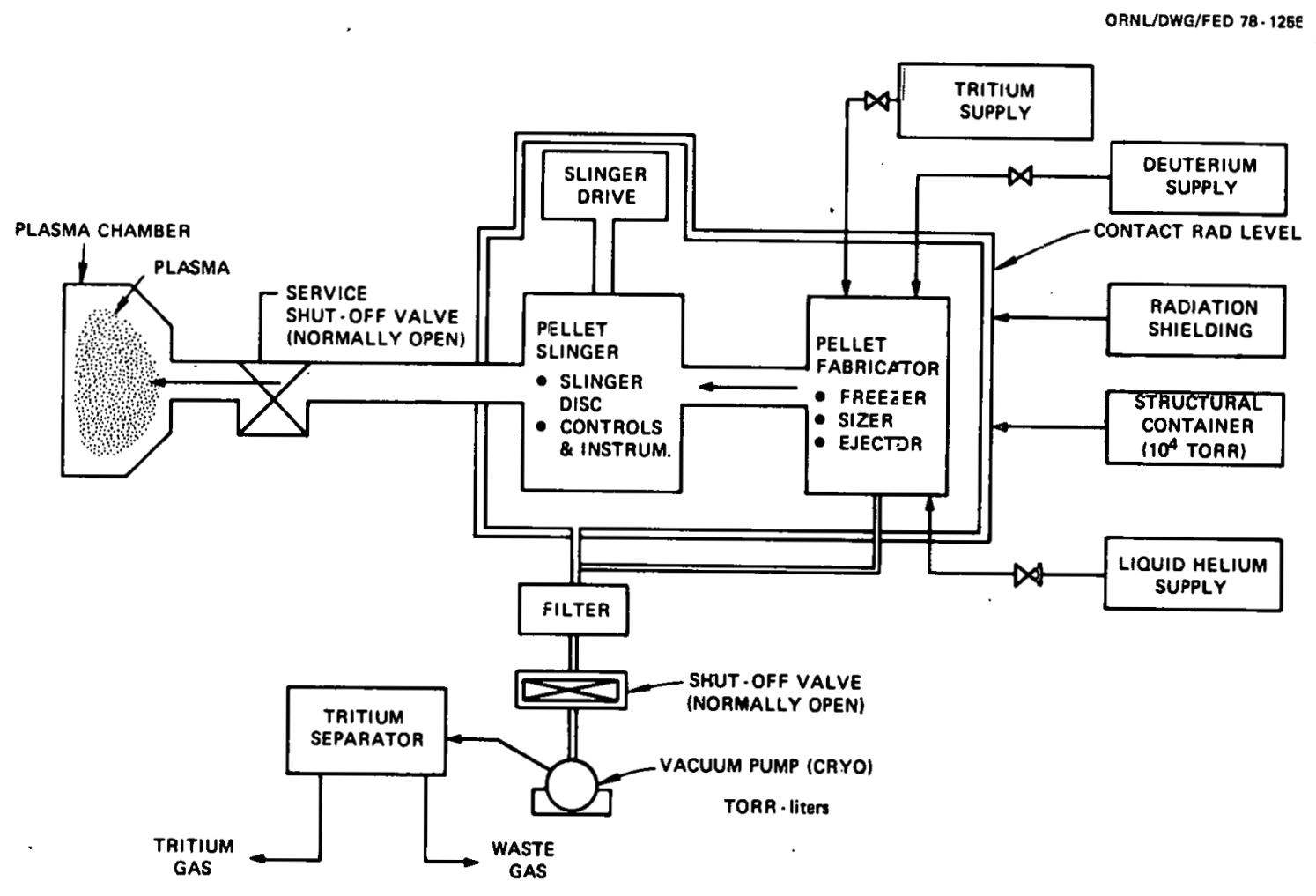

Fig. 4.1. Fuel pellet injector system. 
THIS PAGE

WAS INTENTIONALLY

LEFT BLANK 



\section{NEUTRAL BEAMS}

\subsection{TECHNICAL NEEDS ASSESSMENT}

\subsubsection{Introduction}

Neutral particle injection is to be used in tokamak fusion devices to reach the high temperatures desired for physics studies in the vicinity of ignition and for ignition in reactors. Ohmic heating $(\mathrm{OH})$ techniques are not expected to provide ignition temperatures in plasmas and must be assisted by neutral beams (and/or by rf heating, if it is shown to be feasible).

Previous considerations pointed to the expected need of high energy beams to penetrate to near the plasma center. Present calculations seem to support the systems advantages of using injection scenarios that cal1 for moderate energy (100- to 150-keV) beams. This avoids the use of high power neutral beams with particle energies greater than $200 \mathrm{keV}$, which are not readily available. The efficiency of such high energy, positive ion source beam 1 ines becomes quite low $(<20 \%)$ and requires large quantities of power. Negative ion sources, which have the potential for greater efficiencies, are in the early stages of development and are five years or more behind the positive ion sources in possible applications. The system needs growing out of these data have provided the impetus to evolve injection scenarios which may avoid the need for high energy beams, such as small radius startup, low density, full radius startup, etc.

Direct recovery of the energy in the unneutralized particles in a beam line is being developed and is considered a part of the TNS Reference Design. Such use is expected to allow the achievement of power efficiencies over $50 \%$ in the 120 - to $150-\mathrm{keV}$ positive ion source beam rines. The TNS Reference Design neutral beam system is described in Sect. 5.2. 
5.7.c Status of System and Equipment Development anc Ava ijability

Five key elements of the neutral injection system have been selected. This discussion evaluates their status and readiness for fabricability and reliable, cost-effective operation.

The key elements (see Fig. 5.1) are:

(1: tre ion source,

(2i) the fower supplies,

(3i) the vacuum components and pumping system,

(4i) thie controls and diagnostics, and

(5i) the direct recovery system.

1.

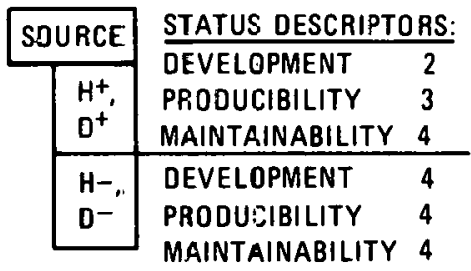

2.

\begin{tabular}{|c|}
\hline POWER \\
\hline SOURCE AND \\
ARC SUPPLIES \\
\hline ACCEL \\
SUPPLIES \\
\hline
\end{tabular}

STATUS EESCRIPTJRS: \begin{tabular}{ll}
\hline DEJELOPMENT & 1 \\
PRODUCIBILITY & 2
\end{tabular} MAINTAINABILITY 3

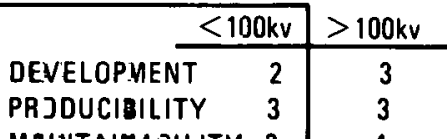

3.

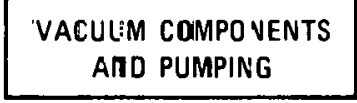

STATUS DESCRIPTORS:

\begin{tabular}{ll}
\hline DEVELOPMENT & 1 \\
PRDJUCIBILITY & 2 \\
MAINTAINABILITY & 3
\end{tabular}

4.

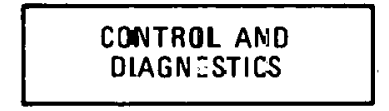

STATIUS DESCRIPTORS: OEVELOPMENT 3 PFODUCIBILITY 3

MAINTAINA3ILITY 2

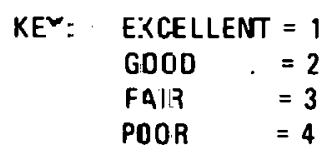

5.

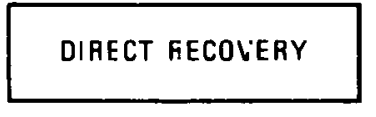

\begin{tabular}{lc}
\multicolumn{3}{l}{ STATUS DESCRIPTORS: } \\
\hline DEVELOPMBNT & 3 \\
PRODUCIBILITY & 4 \\
MAINTAINABILITY & 4
\end{tabular}

Fig. E.1. Status of key elenents of neutral bear injection system: 
The source is further divided into two options, a $\mathrm{H}^{+} \mathrm{D}^{+}$sjurce and a $\mathrm{H}^{-} \mathrm{D}^{-}$source, even though the TNS Reference Design now shows the positive ion sources. The power supplies can be divided - nto two groups: those for the arc and source and those for particle acceleration. The drift tube is considered with vacuum components and punping. The area of controls and diagnostics is considered of equal importance with the other elements, especially in the context of multiple injection operation. Direct recovery, which will play a key role in the TNS neutral injection system, is also deemed important enough for seprarate evaluation. The status descriptors used in the figure are defined as follows:

- Development refers to the state of scientific and technical feasibility.

- Producibility refers to how ready and suitable the design concept is for detailed design and quantity fabrication of a reliable device.

- Maintainability refers to how well the potential design concept would perform under the remote installation and maintenance procedures dictated by TNS operation.

These status descriptors are general and therefore somewhat overlapping. However, it is felt that they can serve as an aid to an orderly consideration of unsolved problems and to the determination of development needs.

The key technical needs that appear necessarji from this evaluation and from other studies and considerations include:

(1) Beam line development for higher currents (>100 A): longer pulses (>10 sec), and better species contral $(90 \% / 10 \%)$ with positive ion sources at energies of $\leqslant 150 \mathrm{keV}$.

(2) Direct recovery techniques with efficiencies of $80 \%$.

(3) Controls and diagnostics for multiple beam systems.

(4) Developments for cost-effective, reliable oseration and ease of fabrication and maintenance.

It seems evident, relative to the last item, that an increased effort or new effort is required to seriously consider how these elenents and the 
entire system can be developed to be less complicatec and expensive and more reliable, producible, and maintainable. Such an activity would be very valuable in providing active feedback to the development and concepitual design efforts.

\subsection{PROPOSED NEUTRAL BEAM HEATING SYSTEM}

\subsubsection{Function}

The bulk heating system raises the tckamak plasma temperature from that achieved by $\mathrm{rf}$ assisted ohric heating to the ignition level. For this purpose, neutral beam injection has been choser. The system parametars are listed in Table 5.1.

\subsubsection{Design Requirements}

The design requirements for the bulk heating system call for a total injected power of $50 \mathrm{MW}$ with a beam energy of $150 \mathrm{keV}$. The pulse length is $\leqslant 6 \mathrm{sec}$ and the duty cycle is $1 \%$. The system is designed to last for the 10-year lifetime of the machine.

\subsubsection{Design Description}

Because $\mathrm{OH}$ techniques alore are not adequate to provide ignition temperatures in plasmas, they must be assisted by neutral beams and/or by $r f$ heating. The Reference Design uses four beam lines, with three scurces ser line, injecting through four rectangular apertures into the plasma. Each beam line includes a positive ion source, a nectralizer ragion, a direct energy recovery system, and a drift region to connect the beam line to the torus. The total eiectrical efficiency of the beam 1 ine is estimated to be $51 \%$. For the Reference Design 27 asma, a ful1 bore, low density startup is faasible. This scenario raquires injecting 50 . MW of 150-keV beams into the plasma for approximately $6 \mathrm{sec}$. Direct recoveryi of the energy in the unneutralized particles in a beam line is teing developed. This technique should allow the achievement of efficiencies higher than $51 \%$ in the $150-\mathrm{keV}$ positive ion scurce seam 1 ines. 
Table 5.1. Bulk heating system parameters

\begin{tabular}{ll}
\hline Structural/configurational & 3 \\
Sources per beam line & 12 \\
Total sources & 4 \\
Number of beam lines & $0.4 \mathrm{~m} \times 1.2 \mathrm{~m}$ \\
Size of beam apertures into torus & $\geqslant 16^{\circ}$ (in the direction \\
Injection angle (perpendicular to & of pl.asma current) \\
plasma surface) & \\
Performance & $100 \mathrm{~A}$ \\
Source current & $3.9 \mathrm{MW}$ \\
Full energy power/source & $0.4 \mathrm{MW}$ \\
Half energy power/source & $4.3 \mathrm{MW}$ \\
Total power/source & \\
Total full energy injected power & $46.8 \mathrm{MW}$ \\
capability & $51.6 \mathrm{MW}$ \\
Total injected power capability & $101 \mathrm{MW}$ \\
Total power for particle acceleration & $150 \mathrm{keV}$ \\
Beam energy & $0.90 / 0.10$ \\
Full/half energy & \\
Neutralization fractions & \\
(full energy/half energy) & $0.33 / 0.70$ \\
Geometric transmission & 0.88 \\
Pulse length & $\leq \leq 6 \mathrm{sec}$ \\
System power efficiency & $51 \%$ \\
\hline
\end{tabular}


THIS PAGE

WAS INTENTIONALLY

LEFT BLANK 



\section{POLOIDAL FIELD SYSTEM}

\subsection{TECHNICAL NEEDS ASSESSMENT}

The PF system for the TNS Reference Design is described in Sect. 6.2. The selection of the systems approach and of the elements in the system was guided largely by the desire to minimize necessary developments. The key elements that make up this system are:

(1) the $r f$ preheating system,

(2) the superconducting exterior $\mathrm{OH}$ and equilibrium field (EF) coils,

(3) the interior copper EF coils,

(4) the power supplies, including

- transformers,

- 12-pulse rectifier/inverter (thyristor bridges),

- power supply controller, and

- auxiliary equipment,

(5) the motor-generator flywheel (MGF) sets, anc

(6) the electrical distribution systems.

The last three elements have many similarities to state-of-the-art equipment and to equipment being supplied to TFTR, Doublet III, and JET. Therefore, no significant R\&D is considered necessary to ensure feasibility or to determine a proof of principle. However, this is not the case for the first two items.

The 120-GHz, 1-MW rf preheating system can be considered an extrapolation from the technology advancement of the $28-\mathrm{GHz}$ system being developed by Varian Associates for the ELMO Bumpy Torus (EET) program at ORNL. The specific R\&D needs of the TNS rf system are discussed in detail in Sect. 10.1. Theoretical physics calculations of the rf preheating option show a promise of reducing the required startup voltseconds by $18 \mathrm{~V}-\mathrm{sec}$ and the probability of increasing startup time from 2 to $6 \mathrm{sec}$. This has significant ramifications in both the cost and the complexity of the device. The necessary substantiation of these calculations is discussed in Sect. 2. Without rf preheating (or some other less well-defined physics approach) to allow the longer startup time, 
the PF system cost is expected to increase by $\$ 85$ million ( $217 \%$ of total device $\cos t$ ), with the add:tional complication that an interruption scieme will be required. The capability for an extended turn will also be significantly impaired by the absence of $r f$ as the startup requirements consume more volt-seconds. Finally, by permitting the use of a lower plasma loop voltage, $r f$ preheating reduces the requirement of electrical breaks in the plasma vacuum chamber and further simplifies the cevice construction.

Los Alamos Scientific Laboratory (LASL) is plarining the development of a 20-MJ superconducting pulsed energy storage coil to operate in a full bipclar mode and swing fron $7 \mathrm{~T}$ to $-7 \mathrm{~T}$ in $1 \mathrm{sec}$. This field swing rate $(\dot{B} \sim 14 \mathrm{~T} / \mathrm{sec})$ is much more stringent than the $\dot{B} \sim 1.33 \mathrm{~T} / \mathrm{sec}$ sFecifiec in the TMS Reference Design. Although the coil energy of the LRSL coil is significantly lower (20 MJ vs $750 \mathrm{MJ}$ ), the energy is a reflection of the coil size and should not impact the feasibility of the concept. The remaining ccil paraneters, such as eddy current loss, refrigeration, total current, and mechanical stresses, will also be addrassed by the LASL program. Considerations involving the generation of design data on cryogenic properties of structural and insulation materials will be developed in the course of the work being ferformed on beth the LASL program and the ORNL Large Coil Program (LCP).

The ISX-B program being performed at ORNL incorporates the same coil use philosophy and is considered to be the experimert from which the overall PF system approach $w^{\div} 11$ be demonstrated and the proof of principle substantiated.

\subsection{PRGPOSED POLOIDAL FIELD SYSTEM}

\section{E.2.1 Function}

The PF system (1) induces a current in the prasma, (2) maintains the plasma columns in equilibrium both horizontally and vertically, and (3) protects the superconducting TF coils from magnetic flux changes. The parameters of the PF system are shown in Table 6.1. 
Table 6.1. PF system parameters

\section{Primary $\mathrm{OH}$ coil \\ Gereral}

Life, $t_{L}$

Duty cycle, dc

Inside radius of solenoid, $R_{I}$

outside radius of solenoid, $R_{\text {os }}$

Length of central core, $L_{S}$

Number of turns (total), Noh

Mechanical

Maximum hoop stress, $\sigma_{s}$

Electromagnetic

Ampere-turns

Maximum field at winding, $B_{\text {smax }}$

Field at coil axis, $B_{\text {sax }}$

Induced plasma current

(by $\mathrm{OH}$ system), $\mathrm{I}_{p}$

Maximum rate of flux change

in the $\mathrm{OH}$ coil, $\dot{B}$

Coil swing time (full forward

to zero current), $t_{s}$

Coil volt-seconds ( $\mathrm{OH}$ system)

Stored energy, $W$

Coil voltage (maximum), $v_{C}$

Maximum coil current/turn, I

Conductor

Current density overal1, $J_{O A}$

Self-inductance (single turn), $\mathrm{L}_{00}$.

Thermal

Conductor coolant

Total refrigeration required, $P_{\text {ref }}$
10 years

$95 \%$

$0.875 \mathrm{~m}$

$1.13 \mathrm{~m}$

$10 \mathrm{~m}$

2771

20,000 psi

64 MAT

$8 \mathrm{~T}$

$8 \mathrm{~T}$

$4 \mathrm{MA}$

$1.3 \mathrm{~T} / \mathrm{sec}$

$6 \mathrm{sec}$

$5.3 \mathrm{~V}-\mathrm{sec}$

$1.1 \mathrm{GJ}$

$25 \mathrm{kV}$

$23 \mathrm{kA}$

$\mathrm{NDTi}$

$2.5 \mathrm{kA} / \mathrm{cm}^{2}$

$0.37 \mu \mathrm{H}$

Helium

$\mathrm{TBD}^{a}$ 
Table 6.1 (continuec)

\begin{tabular}{ll}
\hline EF coil & \\
General & \\
Lifetime, $t_{L}$ & 9 years \\
Duty cycle, dc & $94 \%$ \\
Number of coil systems, Nsh & 6 \\
Electromagnetic & \\
Ampere-turns & $41 \mathrm{MAT}$ \\
Coil volt-seconds & $24 \mathrm{~V}-\mathrm{sec}$ \\
Stcred energy, $W$ & $2.6 \mathrm{GJ}$ \\
Current/turn, Iac & $23 \mathrm{kA}$ \\
Conductor material & \\
Normal conductor & $\mathrm{Cu}$ \\
Superconductor & $\mathrm{HbTi}$ \\
\hline
\end{tabular}

$a_{T B D}=$ to be determined.

\subsubsection{Design Requirements}

The PF system has a 10-year design lifetime for the main components and allows remote maintenance and replacenent of the coils. Operating with rf preheating, the $\mathrm{OH}$ and $\mathrm{EF}$ coil systems will be capable of supplying $53 \mathrm{~V}$-sec during startup, inducing a plasma current of up to 4 MA. Without rf preheating, $70 \mathrm{y}-\mathrm{sec}$ will be required. The 0 it system will also have sufficient volt-seconds capacity to provide a relatively long burn pulse, which would be adequate for viable reactor ojeration. The EF coils will supply sufficient vertical field to hold the plesma current in equilibrium during the burn phase.

\subsubsection{Design Description}

An air core, hybrid PF sysiem incorporating rf assisted startup was selected for the FY 1978 Reference Design. The PF system consists of a suserconducting $\mathrm{OH}$ cail, interior (inside the TF coil bore) normal cosper EF coils which carry $35 \%$ of the EF current, and exterici (outside the TF coil bore) supercondscting EF coils which carry $65 \%$ of the EF 
current. The name "hybrid" is used to identify the mix of interior and exterior EF coils.

The size of the poloidal field bore for the Rəference Design was taken from system studies which identified the geometric characteristics (i.e., the plasma size and major radius) for a minimum cost ignited tokamak reactor. ${ }^{1}$ The $\mathrm{OH}$ system was sized for maximum utilization of the poloidal bore and for operation at a maximum field of $3 \mathrm{~T}$. The PF system will supply $83 \mathrm{~V}$-sec when operating with a half-biased $\mathrm{OH}$ system swinging from $+8 \mathrm{~T}$ to $-8 \mathrm{~T}$, which is adequate for startup and $500 \mathrm{sec}$ of burn.

Placement of the EF coils is important from the standpoint of maintaining plasma equilibrium and shape during the heating and burn phase, influencing power consumption, protecting the TF coils against plasma disruption, and servicing the coils. In the Reference Design, the EF coil system consists of six coil systems, named primarily for their locations with respect to the TF coil bore and their rough proximity to the plasma: interior inside coils (II), interior D-shape coils (ID), interior outside coils (IO), exterior inside coils (EI), exterior D-shape coils (ED), and exterior outside coils (EO). The PF system is shown in Fig. 6.1. As noted, the EF coils inside the TF coil bore are normal copper and those outside are superconductinc. This arrangement of interior and exterior coils can maintain the plasma position and D-shape for a wide range of beta values.

Because of the intrinsic engineering difficulties with the assembly and repair of interior coils in the radioactive environment of a D-T tokamak reactor, an effort was made to reduce the number of turns of these coils. The $65 \%-35 \%$ split between exterior and interior EF coils offered a reasonable balance between coil maintenance, TF coil protection, and power requirements.

The time behaviors of the EF and $\mathrm{OH}$ coil currents are shown in Fig. 6.2, based on $r f$ preheating during startup. The $r f$ assisted startup was chosen because a substantial reduction is realized in the startup loop voltage and resistive volt-second losses, along with an increase in the startup time relative to operating without a $r f$ preheating system. The $r f$ preheating is supplied by five 200-kW gyrokl:/strons, which deliver 1 MW to tile plasma during the 6-sec startup period. 


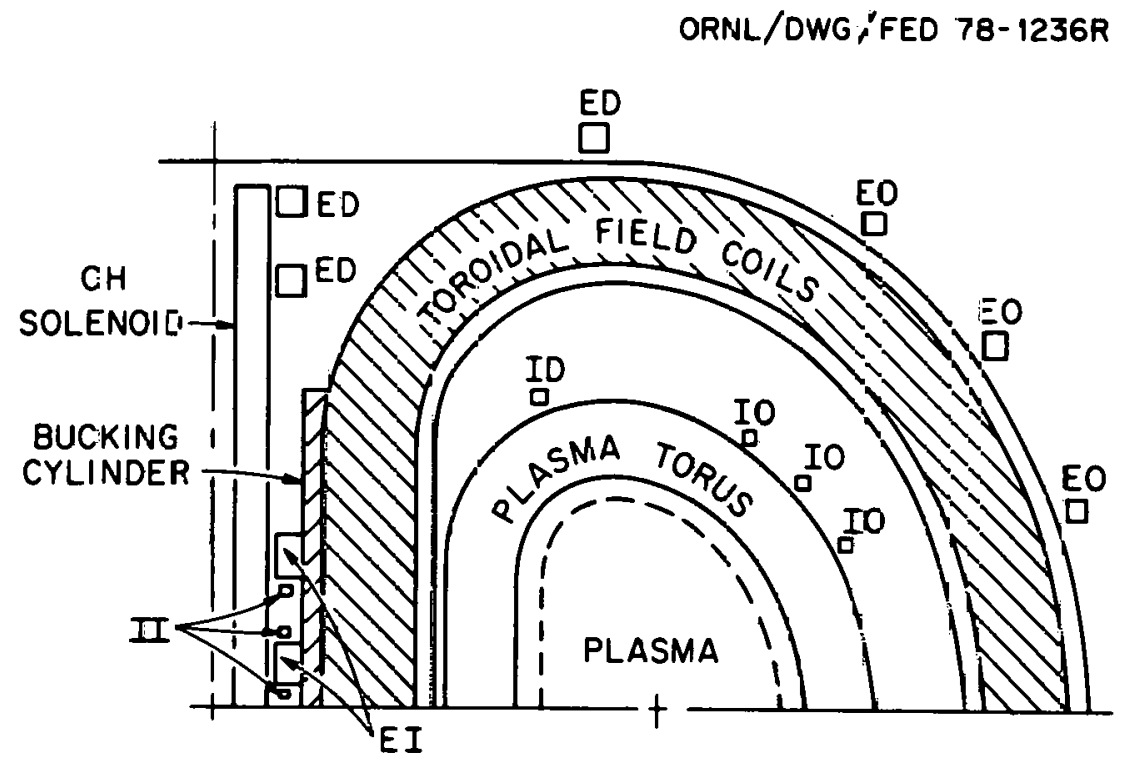

Fig. 6.1. PF coil locations. 
ORNL/DWG/FED 78-1237R

\section{CURRENT WAVE FORMS}
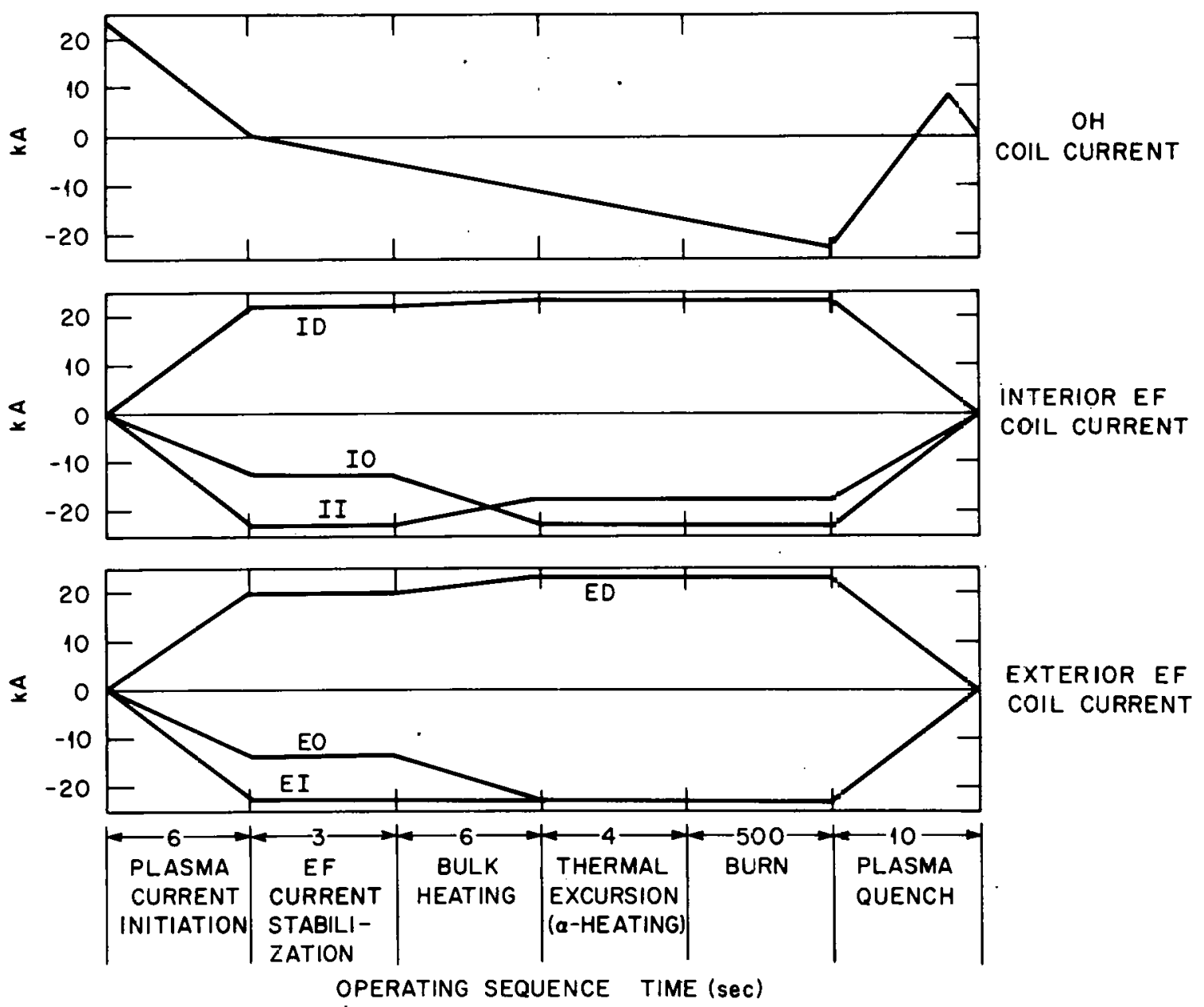

Fig. 6.2. PF coil current waveforms.

\section{REFERENCE}

1. TNS Engineering Staff, Four Ignition TNS Tokamak Reactor Sustems Design Study, WFPS-TME-07i, Westinghouse Electric Corporation, Pittsburgh, Pennsylvania (October 1977). 
THIS PAGE

WAS INTENTIONALLY

LEFT BLANK 


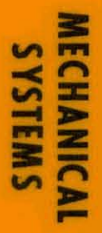




\section{MECHANICAL SYSTEMS}

\subsection{TECHNICAL NEEDS ASSESSMENT}

\subsubsection{Introduction}

One of the TNS objectives, as stated in Ref. 1, is to provide a near-term means of focusing the efforts of the national iusion program, which seeks an economically viable fusion reactor concept. In this light, the assessment of the TNS mechanical systems has four considerations:

(1) What are the tasks to be done?

(2) Are those tasks in place; that is, does a technology base exist?

(3) What are the time frames?

(4) Does this represent a go or no-go situatian regarding the total device feasibility?

The following generalizations can be made about mechanical systems as we examine each of the four considerations.

\section{Tasks to be done}

There are four major tasks to be accomplished:

- Define the mechanical/structural loads on all major components.

- Establish the operating environment of the major components.

- Define the useful life of the total machine and of its components.

- Design the mechanical systems to fit these criteria.

\section{Technology base}

Based on the current understanding of tokamass and advanced reactor design studies to date, the technology base for these tasks does exist. Although it may be argued that the materials technology is lacking or that certain dynamic load phenomena are not fully understood, these objections really represent design obstacles which prevent an optimum 
configuration: they force the configuration to be comoromised in terms of Lseful life (i.e., number of pulses, neutron fluence, operating temceraturı, etc.).

\section{Time frames}

The time frames to overcome these design obstacles appear to be in place. A rigorous fusion materials program is under way. A veriety of results from Doublet III, ISX-B, PDX, PLT, and TFTR, as well as data from the Tritium Systems Test Assembly (TSTA) and LCP, will be available in time for a TNS start date in 1984.

\section{Go or no-go situation}

$A$ go situation exists for the start of TNS from a mechanical systems point of view because a viable technology base is in place. Optimization of the tokamak configuration is a mechanical design coal.

Agair, these comments are generalizations for tre R\&D needs of mechanical systems. The following discussions for the vacuum vessel and limiters, machine structure, remote servicing/device disassembly, and shialding discuss ir detail the four R\&D considerations.

\subsubsection{Toroidal Vacuum Vessel}

A sufficiert technology base exists for the design of the plasma chamber, and existing fabrication technology is adequate to construct such a vessel. Several areas, however, are not well understood and will result in conservative design criteria, detracting from an optimum reactor design. They are:

* plasma disruptions,

- allowable impurity levels,

- limiter materials, and

- materials behavior in a high temperature, high neution flux environnent. 
Plasma disruption phenomena are just beginning to be studied for reactor-like power levels. The solution appears ts be in controlling these dynamic thermal and mechanical loads through instrumentation. Other areas in need of further understanding or design experience have technology programs in place. They include the following.

- Data for radiation damage and mechanical properties at elevated temperatures for candidate materials are expe:ted from fission reactor materials programs, and specific data will be generated from the fusion materials test programs, incl dding TNS.

- Data for verification of materials performance under plasma conditions will be generated from ISX, PDX, P_T, and TFTR.

- Selection and subsequent demonstration of strictural dielectric materials are expected from TFTR.

- Design and development experience for mechanical seals in lieu. of welding for vessel penetrations (e.g., diagnostics) is expected from TFTR.

\subsubsection{Machine Structure}

The technology base exists for the design and fabrication of the overall machine structure. However, several areas require further development in order to achieve design optimization. These are:

- The mechanical properties of materials at cryogenic temperatures. This area is to be addressed by the National Bureau of Standards (NBS) at Boulder, Colorado.

- Radiation effects on materials properties at elevated temperatures. This area is being studied in materials test programs sponsored by the Department of Energy's Office of Fusion Energy and in fission reactor programs.

- Development of analytical methods for induced transient loading on structure and components. Liaison with the TFTR final design is required. 
- Structural design criteria. While not necessarily a development need, these criteria require consideration before and during conceptual design. Definition of loading conditions, margins of safety, environmental safety, etc., will form the basis =or design. Lack of established criteria will penalize the cesign with costly conservatism. Primary structural tests are not envisioned, provided the configuration evolves into a structurally determinant arrangement. National safety standards and criteria do not exist for these fusion sys.tams but should be developed in the process of engineering TNS.

For a TNS to be built in the next 12 years, there are adequate structural materials. Because of this near-term goal, development and production of any new materials or alloys are unrealistic. Thus, the materials choices are austenitic stainless steels ard perhaps some al uminum all jys.

\subsubsection{Remote Servicing}

The technology base for remote servicing concepts and equipment exists and is sufficient to support a INS design. This technology is derived from the nuclear fission industry and the aerosface community and requ'res same adaptation for the unique problems of tokamak servicing. In particular, further development of remote handling ccincepts and equipment is needed to oftimize device availability.

Several inputs are expected to support TNS, chiefly; the TFTR development and demonstrat: on during the TNS conceptual design period and the JET design experience through 1980-81. It is essential that TNS establish its cwn program of remote systems verification after the TNS conceptual design is completed. This can be accomp ished with a series of mock-up systems, to be completed before a final design review. Specific areas to be investigated are handling of large components, remote cutting and welding, leak detection, and vieving systems. This TNS development program, coupled with liaison to ongoing TFTR development and demonstration, will provide a sound basis for the device design. 


\subsubsection{Shielding}

The existing funded R\&D programs for TNS provide an adequate base to initiate conceptual design. Uncertainty in the shield system desigri criteria will principally affect device cost (siza), not schedule. However, if active impurity control requires a divertor, development of acceptable shielding for the divertor penetration could affect the present schedule. TNS final design will require some modifications and changes in emphasis; however, the basic R\&D programs are in place. The development of a well-defined nuclear data base, substantiated with CTR-focused cross-section data experiments, suitable computational tools (including codes and input data), and an experimental program to validate calculated results, is essentially under way to provide a basis for optimizing shield design.

The CTR data base currently being assembled is generally focused on the needs of the fusion community and should be assessed with regard to specific TNS requirements. Radiation transport computer codes exist but will require some modification for application to toroidal geometry and for solution of radiation streaming. No suitable code exists for the calculation of accurate distributions of the radioactivity and afterheat; an evaluated activation data library and codes must be developed to access these data efficiently. These calculations are presently performed in many disjointed steps, and increased efficiency and shield optimization will result from an automated procedure. Integral experiments to study the materials and configuration of the TNS shield are funded and under way at ORNL. Future experiments to measure neutron streaming through penetrations in the shield are planned, and these studies will be consistent with the TNS design.

Timely judgments of both physics and technology understanding will be required throughout the design phase to provice design criteria for the shield system. Physics understanding will be required to provide design criteria for total plasma power, power distributions, and shield eddy currents. Impurity control R\&D programs could identify additional requirements for the shield system if a divertor (with a itendant penetrations) is found to be necessary. This physics input is closely 
interfaced to our understanding of plasma transport fhenomena and scaling law's and will not be forthcoming from a single experiment. Ra:her, evaluations of all key physics expariments and analytic developments will be an orgoing process and will be factored into the shield R\&D program; such evaluations will be a topic for each of the scheduled design reviews.

Technology understanding from other R\&D programs will be required to srovide design criteria for $T F=0 i 1$ heat input, hec.t removal capability, anc vacuure vessel penetrations. The Large Coil Segment (LCS) and LCP R\&[1 programs are scheduled to provide relevant data on TF coil heat trensfer characteristics. Consideration of the impact of penetrations on shield effectiveness will be closely interfaced with the neutral beam anci diagncstics programs.

\subsection{PROPGSED MECHANICAL SYSTEMS}

\subsubsection{Limiter System}

\section{Function}

Limiters provide physical protection to the first wall during uncontrolled plasma stértup or shutdown. In additior, they can provide pläsma shaping as a scrape-off mecnanism. Limiters cre either fixed or movable and either actively or passively' cooled.

\section{Design description}

The lim:ter system is made up of two sets of poloidal limiters, located $180^{\circ}$ apart. Eäch set consists of four fixed units, as shown in Fig. 7.1. Due to the irregular shape of the torus cross section and the fact that the limiters are for first wall protection only, and not plessma shaping, fixed units were chosen.

A passively cooled liniter is assumed. An actively cooled system is avoidec because of (1) the added complexity of sufplying coolant to the limiter and (2) the possibility of coolant leakirg to the plasma in the event of a limiter coolant tube burnout. 


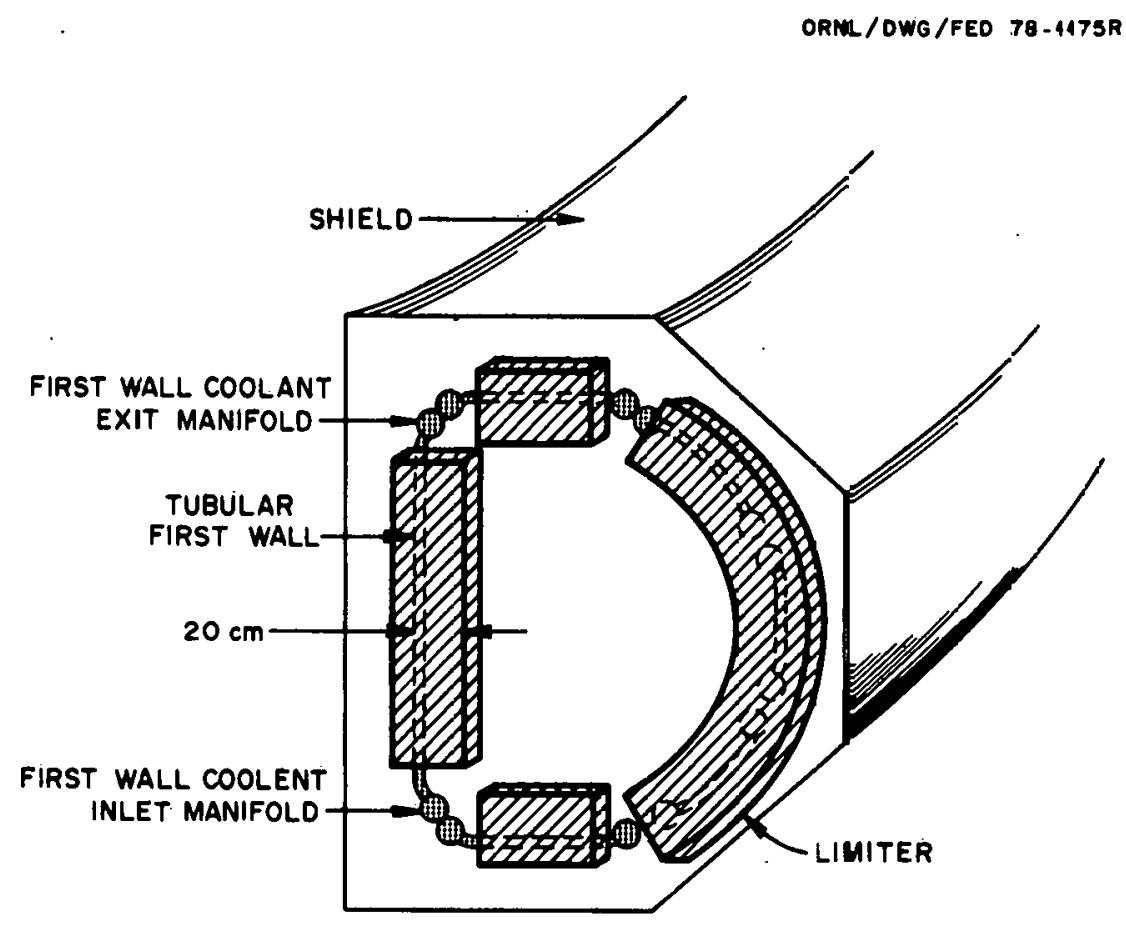

Fig. 7.1. Poloidal limiter.

Graphite is the limiter material; its low- $Z$ sharacteristics are desirable in regard to limiter contributions to plasma impurities. Graphite's thermal properties (high melting point, shock resistance, ard low expansion) are all suitable for the operating environment. The graphite panels, which are 1-2 cm thick, are installed between adjacent first wa 11 modules and extend $20 \mathrm{~cm}$ from the first wall toward the plasma. It is intended that they last for the life of the first wall. Their replacement would be part of the scheduled naintenance of the first wal1. Limiters can be removed only by disassembling the torus.

\subsubsection{First Wall}

\section{Function}

The primary function of the first wall, in conjunction with the divertor, is to absorb and remove the energy associated with the alpha particles; which makes up approximately $20 \%$ of the total plasma energy. 
The first wall is designed to take all the alpha particle energy. However, the actual heat load to the first wall is estimated to be equivalent to $50 \%$ of the alpha particle energy with the remaining $50 \%$ handled by the bundle divertor. Material damage assaciated with particlesurface interactions (e.g., sputtering, embrittlement, etc.) is taken by the first wall. The parameters of the first wall are 1 isted in Table 7.1.

Table 7.1. First wall parameters

\begin{tabular}{|c|c|}
\hline Construction & $\begin{array}{l}\text { 2.5-cm-diam stainless steel } \\
\text { tubes }\end{array}$ \\
\hline Yaximım heat Joad (design value! & $226 \mathrm{MW}$ \\
\hline Coclant & Water \\
\hline Inlet temperature & $40^{\circ} \mathrm{C}$ \\
\hline Exit temperature & $\sim 750^{\circ} \mathrm{C}$ \\
\hline Actual heat load & $113 \mathrm{MW}$ \\
\hline Neutron wall loading, $L_{W}$ & $2.38 \mathrm{MW} / \mathrm{m}^{2}$ \\
\hline Yaximum wall loading (sumface heat) & $0.60 \mathrm{MW} / \mathrm{m}^{2}$ \\
\hline Actual wall loading (surface heat) & $0.30 \mathrm{MW} / \mathrm{m}^{2}$ \\
\hline Lifetime & $\begin{array}{l}4.5 \times 10^{5} \text { cy }=1 \text { es }(10 \text { years } \\
\text { operation) }\end{array}$ \\
\hline
\end{tabular}

\section{Design description}

Tyзe 316 stainless steel tubes, $2.5 \mathrm{~cm}$ in diameter, are brazed to type 315 stiffened sheet steel to make up modular first wall panels (see Fiy. 7.?). Each of the faceted inner surfaces of the plasma chamber is lined with an indeperidentlif cooled panel. The panels are mechanically fastened to the chamber so that induced thermal stresses in the panels are minimal. Type 31.6 stainless steel was chosen because its physical properties are adequate to meet the design requirements and because its use presents an opportunity to expand the nuclear/thermal dati base for this materiai. 


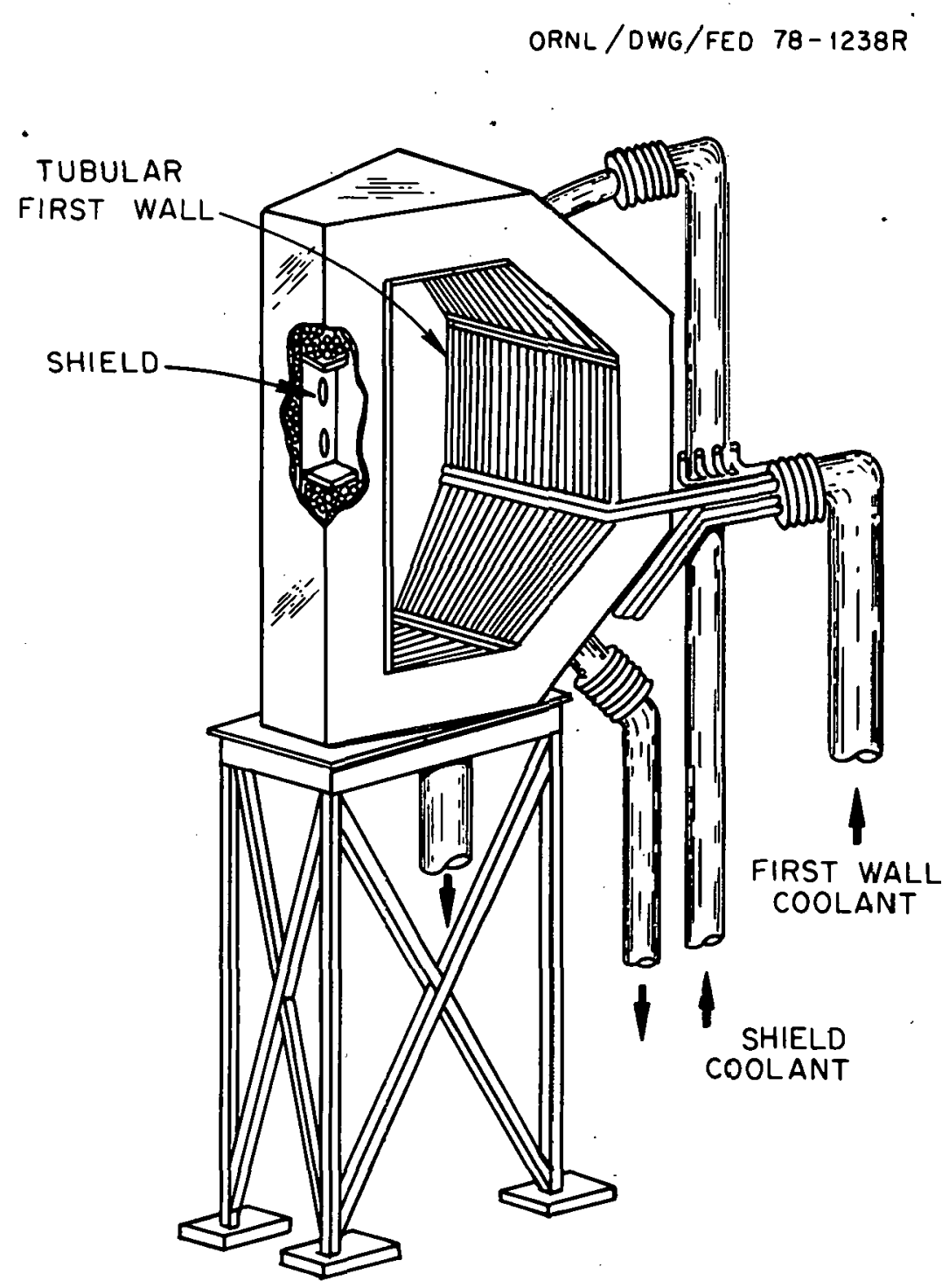

Fig. 7.2. First wall.

The total first wall installation consists of 6 panels for each of the 16 sectors. Sectors may be removed from the davice for replacement or repair.

. The first wall is expected to last for the proposed 10-year life of the facility (based on the mission description in Ref. 2). Adherence to this schedule requires approximately $4.5 \times 10^{5}$ pulses. Tris number is within the low cycle fatigue limit of stainless steel even if the total alpha particle energy is deposited in the first wa 11. The actual heat load to the first wall is estimated to be $50 \%$ of the alpha particle 
energy ( $113 \mathrm{MW}$ ) with the remaining $50 \%$ handled by tree bundle divertor. However, the coolant flow rate through the tabular first wall is adequate to transfer all the alpha particle energy $(2.26 \mathrm{MW})$ at a temperature rise of approximately $110^{\circ} \mathrm{C}$.

\subsubsection{Shield}

\section{Function}

The functions of the shield are to:

(1) reduce nuclear heating in the TF coils to acceptable levels,

(i) minimize activation of reactor components arid auxiliary systems,

(3) attenuate radiation from activated components during naintenance . shutdown periods,

(4) reduce radiation streaming around and throuch penetrations that view tha plasma, and

(E) transfer the heat generated by attenuation of neutron and gamma fluxes by means of an active cooling system.

The shield parameters are listed in Table 7.?.

Tabl $\cong$ 7.2. Shield parameters

\begin{tabular}{|c|c|}
\hline Shjeld material & $\begin{array}{l}\text { Stainless steel balls and } \\
\text { borated water }\end{array}$ \\
\hline Mzjjor radius, $R$ & $5.0 \mathrm{~m}$ \\
\hline Shield minor radius & $\sim 1.5 \mathrm{~m}$ \\
\hline Shjield thickness & $0.6 \mathrm{~m}$ \\
\hline $\begin{array}{l}\text { Shield thickness } \\
\text { (around penetrations) }\end{array}$ & $0.3 .3 \mathrm{~m}$ \\
\hline Nurber of sectors & 16 \\
\hline Coclant & Low presslire borated water \\
\hline Coclant heat load & 122i] MW \\
\hline Nelitron wall loading, $\mathrm{L}_{W}$ & $2.4 \mathrm{MW} / \mathrm{m}^{2}$ \\
\hline
\end{tabular}




\section{Design requirements}

The shield between the first wall and the TF coils will be thick enough so that the total time-averaged nuclear heating rate in the superconducting TF coils will be $\leqslant 20 \mathrm{~kW}$ (about one-third of the total pulse average heating rate in the TF coils).

\section{Design description}

The D-T plasma operation will produce large quantities of 14.1-MeV neutrons. These high energy particles, plus the secondary radiations they generate in the form of gamma rays and low energy neutrons, will cause damage to materials and represent a hazard to personel. The sinielding system of a D-T fusion device normally includes four components: (1) blanket, (2) bulk shield, (3) penetrat on shield, and (4) biological shield. The primary function of the blanket and bulk shield is to convert the kinetic energy of fusion neutrons and secondary gamma rays into heat. The additional function reserved for the blanket is breeding tritium; since TNS, at this time, does not include fuel generation, we are describing only a bulk shield héving a dual purpose, energy conversion and reactor component protection by neutron attenuation. The penetration shielding surrounds the ducts for neutral beam injectors, divertors, vacuum pumps, fueling, and diagnostics. It provides virtually no attenuation for direct streaming but will protect components (such as the TF coils) that are adjacent to the penetrations. The biological shield is generally considered to be the concrete reactor cell, which provides the last barrier for neutron attenuation curing fusion and limits activation levels outside the building to $1 \in \mathrm{ss}$ than $100 \mathrm{mrem} / \mathrm{year}$.

The bulk shield material is stainless steel ir: the form of 1-cm-diam balls with borated water acting as the coolant for each module. The minimum outer shield thickness (i.e., the shielding furthest from the bucking cylinder) will limit the PF coil organic irsulation to a radiation level of $10^{10}$ rads over the life of the tokamak. The mininum inner shield thickness will limit the total time-averaged nuclear heating rate in the superconducting TF coils to an acceptable rate. A 5-cm thickness of lead is part of the bulk shield. 
The total device shielding thickness was evaluated relative to the size and numser of penetrations. The total effectiveness of the shielding in the reactor cell is greatiy influenced by the area of the penetrations into the torus. The penetration shield around the reutra 1 beam duct is $30 \mathrm{~cm}$ thick and the concrete thickness for the reactior cell is $2 \mathrm{~m}$ thick, assuming ordinary concrete.

\subsubsection{Machine Structure}

\section{Function}

The machine s-ructure provides the structural support for the componen:s that make up the tokarak device: the torus plasma chamber, the TF and PF coil supports, the dewar structure, and peripheral components that interface with the torus, suct as the neutra beam injectors, the rf heating syszem, the pellet fueling device, and the divertor.

\section{Design requirements}

The machine s:ructure will be desigred in compliance with accepted ASME safety codes =o ensure a reasonable degree of conservatism and consistency in appiying the design rules. Deviations will be permissible in areas where the codes do not apply anc where revisions or extensions of the codes are warranted. The machine structure will be designed to complement the remote handling characteristics of the overall device, have a 10-year minimum design 1 ife, and incorporate materials that minimize neutron activation and have low magnetic permeability and high eiectrical resistance.

\section{Design description}

The major components that make up the machine structure are listed with corresponding design descriftions (refer to Fig. 7.3 to establish the location of each component within the tokamak device).

Torus plasma chamber. The torus plasma chamber surrounds the plasma, contains the bulk shieid material, and provides suppert for the 


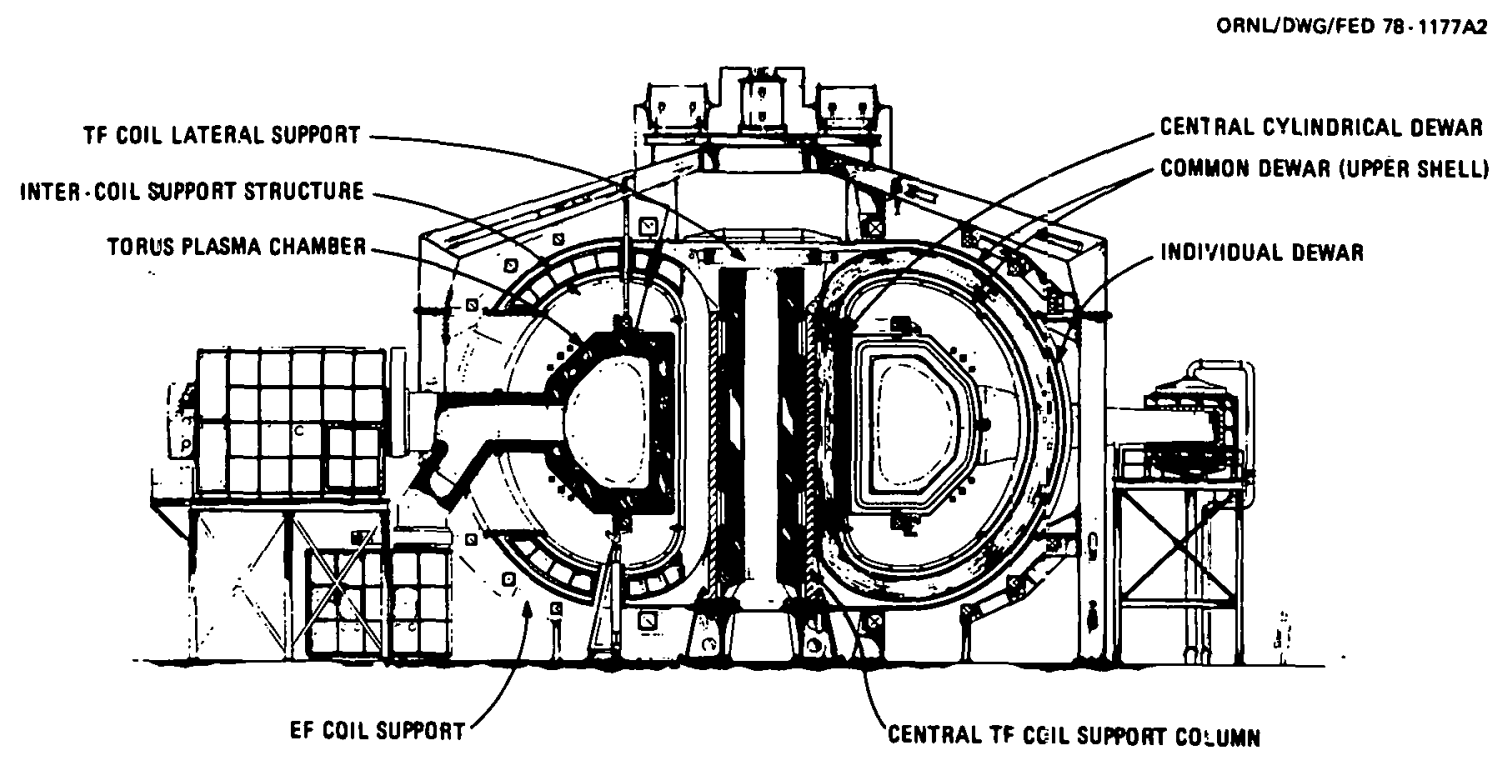

Fig. 7.3. Major components of the machine structure.

first wall. The first wall structure is mechanically supported off the inner wall of the sectors. The bulk shield material, consisting of stainless steel balls and borated water, is located in the interspace of the sector walls. The torus is made up of 16 sectors which are mechanically sealed and bolted at sector interfaces. Each $22.5^{\circ}$ sector has a trapezoidal cross section and consists of three iriner and outer, singlecurvature-formed, stainless steel sheets separated by internal stiffeners and closed at each end. Four sectors form a quadrant $\left(90^{\circ}\right)$ of the torus and are supported from below by a curved beam attached to a truss structure connected to ground. The curved beam and truss structure are configured to allow a torus quadrant to be moved radially outward a small distance to provide initial intersector clearance during sector removal operations.

With a 1116 sectors connected, a rigid structure is formed. Electromagnetically induced, radially outward forces are relatively small and are reacted by the intersector bolts. The interior D-shape coils of the poloidal field system, located at the top and bottom of the torus, act as bucking rings to support any electromagnetically induced centering forces. The torus itself is designed to withstand the 1-atm pressure differential required for shakedown operations. 
Coil support structures. Eentral TF coil support column: The central support column is a cryogenically cold (5K) struciure which (1) transfers tne gravity forces of the TF coils anc intercoil structure to a crycgenically isolated machine support base anc (2) supports a partion of the magnetically induced centering and overturning forces of the IF coils based on the relative stiffness betweer: the support column and the intercoil structure. The support column is a 12-sided polygon with a circular inner diameter. The structure has a vertical groove along the full height of each face to react a portion of the TF coil torsional forces. It is electrically segmented to reduce eddy currents.

Intercoil support structure: The intercoil support structure, which reacts a portion of the magnetically induced TF ccil forces, is a bc $x$ beam construction located between coils and mechanically fastened to tr.e TF cail structure along the upper and lower portions of the coils. Tr:a torsional load is balanced at the machine midplane by differential bending in the outer portion of the TF coil and by keyirg action at the buicking cylinder. A structural insulating shim of glass-reinforced epoxy acts as an interface between the TF coils and the intercoil structure to interrupt the eddy current path. Fault cordition requirements for the failure of a single TF coil and the associated loads have not been defined; therefore, the extent of the intercoil structure required for this conditicn has not been addressed. The design shown irdicates that out-of-plane fault loads generated along the unsupported section of the TF coil are beamed to the existing intercoil structure. If the stresses generated on the TF coil under this concition are unacceftable, it appears feasitle to add local demountatile supports to reduce the stresses without compromising the remote mairtenance features of the device.

EF coil support: The intercoil forces associated with the EF coils are supported by an interconnecting structure between the EF coils. The upper support structure and machine floor support the gravity forces and any imbalance in the coil system.

TF coil lateral support: The TF coil lateral support member is a ring located at the top of the $\mathrm{OH}$ solenoid and connected by pins to the 
TF coils to support the imbalance in the TF coil gravity loads during initial assembly.

Dewar structure. The dewar structure forms a vacuur enclosure for the cryogenically cooled components that make up the tokamak device: the TF coils and intercoil structure, the $\mathrm{OH}$ solenoid, the interior $\mathrm{EF}$ coils, and the central support column. The dewars minimize tritium collection on the surfaces of these components and enable them to maintain their cryogenic condition when the vacuum building is repressurized, eliminating the dependence of the tokamak device on their cooldown and warmup cycles.

The dewar structure consists of two continucus stiffened shells, which enclose the upper and lower portions of the Tf coils; a central cylindrical dewar, which encloses the inboard legs of the TF coils; and individual rectangular dewars, which enclose the outboard legs of each TF coil. The secondary vacuum environment proviced by the vacuum building allows the use of mechanical connections to join the dewar sections.

\section{REFERENCES}

1. Oak Ridge TNS Program Staff, Drabt Program Plan for TNS - The Next Step After the Tokamak Fusion Test Reactor. Part II - R\&D Needs Assessment, ORNL/TM-5983, Oak Ridge, Tennessee (December 1977).

2. D. Steiner, W. R. Becraft, T. G. Brown, W. A. Houlberg, A. T. Mense, Y-K. M. Peng, R. L. Reid, J. A. Rome, C. Sarcella, T. E. Shannon, P. T. Spampinato, W. M. Wells, and G. W. Wiseman, Oak Ridge TNS Program: Summary of FY 1978 Activities, ORNL/TM-6720, Oak Ridge, Tennessee (1979). 
THIS PAGE

WAS INTENTIONALLY

LEFT BLANK 


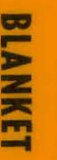




\section{BLANKET}

The TNS Reference Design includes design corcepts for the first wall and methods for shielding. Although complete requirements for a blanket are to be addressed in later design studies, it was decided that these technical needs assessments, since they deal with the future program needs and provide guidance to the R\&D effort, should include all the critical needs of the blanket and shield.

The blanket functions envisioned for a tokamak fusion power reactor include the very important ones of absorbing the energies from the energetic neutrons and from the nonneutron plasma energy deposited on the first wal1, removing the heat via compatible fluids for steam and electric power generation, and breeding and handling tritium.

The impingement of energy on the blanket and its deposition in the blanket are processes that require considerable iterative consideration of composition of materials, nonuniformity of energy flux, cooling approaches and capacities, and the structural proserties of the materials. In addition to the thermal and mechanical stresses due to the normal duty cycle operation, transients, major disruptions, and off-design point performance must be considered so that the design integrity is maintained. The best estimates to date of operation of blanket concepts point to useful lifetimes shorter than those of other major device elements. Thus, replacement of the total blanket or of weakened modules will be required during the useful life span of the fusion power reactor. The many interiocking concerns in selecting a blanket design approach have been categorized into nine areas.

\subsection{NUCLEAR DATA BASE}

The establishment of a well-defined nuclear data base for all materials under consideration is required to provide the means to obtain the desired accuracy in the associated analyses and to permit accurate optimization of the breeding and shielding design. 
The nuclear data base presently available is incomplete and contains large uncertainties. As the evaluations of the designs and their performance mature, the analyses required to support the design will become substantially more sophisticated. The need to know and reduce the lavel of uncertainty $n$ these analyses will increase.

The main problem concerns the accurate description of the nuclear heating and radiation damage in tre blanket, shield, magnets, and structures. In particular, shielcing of superconducting coils at peretrations in the reactor blanket will be a sensitive area requiring accurate data to avoid overconservative and hence ccstly desicn. Other problems include the determination of tritium breeding capability in the exjerimental breeding modules, the description of induced activity in the various structural materials in the reactor, and the requirements for nuclear data on the ijel cycle reaction process and the rediation shielding evaluations.

\subsection{NEUTRONICS METHODS}

The development of neutronics methods for accurate descr"ption of the ruclear performance of finite-sized, realisticaliy configured blankets/shields is required, along with bench-scale tests, to provide a deroristrated nuclear design capability.

Generalized numerical techniques exist to describe sophisticated. space-time nuclear behavior; the Monte Carlo technique is one example. However, methods must be developed on the basis of such techniques and then tested against bench-scale experiments to provide the calculational capability needed for detailed analyses of future designs. This includes the capability to describe the system under analysis in all dimensions, irsluding the effect of penetrations and the proper neutron source term for the toroidal fusing flasma geometry. 


\subsection{THERMAL-HYDRAULIC AND HEAT TRANSFER ANALYSIS}

The development of computational methods to quantify the thermal fluid behavior of promising coolants is required to provide the therma $7-$ hydraulic and heat transfer bases for the design.

The geometry of the coolant system, and of the blanket it is cooling, is likely to be complicated, requiring sophisticated multidimensional calculational methods to describe accurately the thermal fluid and heat transfer behavior of the blanket for normal pulsed operation and for postulated off-design and accident conditions. The impact of the thermal

cycling resulting from pulsed operation is recognized as a potentially severe problem. Accuracy in assessing the thermal stress conditions will be required to define materials development needs.

\subsection{PULSED MAGNETIC FIELD EFFECTS}

A determination of how the design and perfornance of the pulsed magnetic field systems are affected by the proposed mechanical aspects of the blanket and shield must be made to provide a basis for the electromagnetic aspects of the blanket/shield design.

The specific blanket and shield design selected may use many materials of varying conductivities arranged in a complicated configuration. The problem of induced eddy currents (and the resultant forces and fields) has been recognized, and some work has been done in this area. However, electromagnetic field perturbation criteria must be generated, and methods must be developed to describe accurately the electromagnetic effects of nonsinusoidal time variations on the complex blanket and shield designs being considered. Calcdlations of losses in the blanket are also needed to determine the effect of the blanket on the air core volt-second requirement. 


\subsection{ELECTRICAL INSULATION}

Requirements must be established for the electrical insulation of the blanket and shield to provide the basis for the design of any electrical breaks in the bianket.

The blanket and shieid are located in strong electromagnetic fields that undergo large, rapid changes presentiy uncertain in magnitude by much more than a factor of two. Accordingly, criteria for the electrical insulation $o^{-}$the blanket and shield must be develofed. Any special materials required to satisfy such criteria would have to be identified and apprcved, their behavior in a plasma radiation erivironmen: determined, ard their performance under cyclic thermal loads established.

\subsection{LIQUID : ITHIUM CIRCULATION}

Experimental demonstration of the acceptability of circulating liquid 1 thium in the configuration environment is necessary to the qualification of the blarket design.

Liquid lithium has been icentified for use in tokamak power reactors. 1 An understandirg of the performance of circulating liquid lithium in the magnetic field environment must be develcped, so that acceptable performance can be realized.

\subsection{COO_ANT/CONTAINMENT COMPATIEILITY}

The compaibility between candidate coolants and cortainment materials must be determined to develop a basis for prediction of component lifetime.

\subsection{STFUCTURAL MATERIALS}

Supporting R\&D are needed to support the selection of the structural material (including both compasition and metallurgical condition) which 
has optimum end-of-life properties for reactor afplication. This material will form the basis for design of the first wall and other blanket structure.

Radiation effects in the various fusion reactor materials in the first wall and blanket must be known so that a balance can be achieved between conservative (and hence costly) design requirements and shortened working life of the reactor components. The 14-MeV fusion neutrons generated by the thermonuclear reaction will produce significant radiation effects in the first vacuum wall and the structural walls of the blanket. Helium produced by the $(n, \alpha)$ reactions vill embrittle most conventional structural materials, such as type $3 i 6$ stainless steel $(20 \%$ cold worked), which is presently considered the best materials choice for the first experimental reactors. Moreover, rédiation induced damage increases with increasing wall temperature, with a drastic reduction in the creep rupture strength properties of type 316 stainless steel above $550^{\circ} \mathrm{C}$. Uncertainties exist in both irradiation conditions and requirements. Indications from reactor studies consistently support the economic desire for neutron wall loadings high enough to require significant developments.

\subsection{TRITIUM RECOVERY}

A feasible scheme for recovery of tritium fron the blanket must be identified and developed for application to provide a basic design for this essential function in the breeding modules. Significant tritiumrelated questions must be answered before full-scale environmental considerations can be addressed.

The fusion power reactor blanket has been studied in cepth in the ORNL Tokamak Blanket Design Study, ${ }^{2}$ the objective of which was to develop a blanket concept based on near-term technology which appears to satisfy. practical reliability and lifetime needs. An interesting concept has been advanced and preliminary analyses show its promise for application. This very challenging engineering area, impacted by machine complexities and activation that make repairs and changeouts dificult, can be and is being addressed with logical engineering design and analysis. This area 
is of particular va'ue to the engineering test phase, and a continuation of the development is necessary. The requirements in terms of surface anc volumatric heat generation coupled with the large number of power on-off cycles are ciallenging - even if the severe neutron irradiation effects are ignored. The $j$ llowing R\&D activities siould be cf particular bene $=i t$ :

(1) Development of an experimental basis for the concept v:a model testing that includes some limited neutron environment. MHD effects can and sholld be tested in a lithium blanket fluid.

(2) Characterization of the requirements imposed on the blanket (i.e., prart:cle loading and heat loads) from an abnormal or disruption situation as well as a normal cycie.

(3) Continued investigazion of alternate concepts on a relatively lower level.

\section{REFERENCES}

1. W. M. Wells, CRNL Fusion Power Demonstration Study: Lithium as a Blarket Caolart, ORNL/TM-6217, Oak Ridge, Tennessee (Apri1 1978).

2. P. B. Mohr et a 1., Tokamak Blanket Design Studi:: Fy 78 summary Report, 0R.NL/TM-684T, Oak Ridge, Tennessee (19i9). 
을
을
2 


\section{TOROIDAL FIELD SYSTEM}

\subsection{TECHNICAL NEEDS ASSESSMENT}

The major technology needs which affect the status of the TF system in meeting the proposed TNS Reference Design and schedule are discussed here. The system is described in detail in Sect. 9.2.

The Large Coil Program was initiated to advance the design and manufacturing capabilities of organizations that can provide superconducting TF coils of a size and field in the range predicted for fusion needs. A TF coil capable of operating at $10.9 \mathrm{~T}$ extends the capabilities presently being addressed in the LCP; this indicates the need for supplementary emphasis in order to meet the requirement. The tentatively planned development and test programs for conductors suitable for 12-T fields would provide necessary data for the Reference Design. It is expected that the combined results of the LCP coil development and test program and of the 12-T conductor development program would generate sufficient information to provide a proof of principle for the design of the TNS-size TF coil. With the implementation of the 12-T conductor program, it appears that the major R\&D needs associated with a TNS TF coil are being planned in a timely manner.

The application of data from these developments must include many considerations to achieve the desired performance and must establish the confidence necessary to embark on the project design and fabrication. The key objective is to demonstrate reliable operation of a realistic magnet system at selected design conditions without expensive design margins. Success depends on the adequacy of design problem solutions and on the reduction of uncertainties. The specific R\&D emphases needed include:

(1) Production of $\mathrm{Nb}_{3} \mathrm{Sn}$ conductor and manufacture of coils without strain degradation of properties.

(2) Stabilization by boiling helium (degradation of heat transfer flux by vapor blanket). 
(3) Means of coil protection, including the detection of small normal zones, quench pressure in forced flow channeis, and quencr voltage in bath-cooled coils and the control of coil currerts or fault forces.

\subsection{PROPGISED TOROIDAL FIELD SYSTEM}

\section{9.ć.l Fur.ction}

The toroidal field system genarates a toroidal magnetic field that combines with the pulsed poloidal nagnetic field to confine the plasma. The system parameters are listed in Table 9.1.

\section{2̇.2 Design Requirements}

The TF coils must generate a magnetic field on axis $\left(B_{T}\right)$ of $5.3 \mathrm{~T}$ and must have a magnetic field ripple at the plasma edge $\left(s_{a}\right) \cdot 0 f<2.5 \%$ (peak to average). The duty cycle is $100 \%$, and the coil lifetime is 10 years.

\subsubsection{Design Description}

The required on-axis magnetic field of $5.3 \mathrm{~T}$ at a plasma najor radius of $5.0 \mathrm{~m}$ is produced by 12 superconducting TF coi.ts with a horizontal bore of $\epsilon .2 \mathrm{~m}$ and a vertical bore of $9.8 \mathrm{~m}$. This combination of number of coils and coil size achieves a magnetic field ripple of $1.5 \%$ at the plasma edge, which is less than the maximum value of $2.5 \%$. However, these larger TF ccils, relative to the size required for $2.5 \%$ ripple, ailow the PF coils insice the bore of the TF coils to be raised to parking orbits during tcrus segment removal and also c.llow space for the removal of a torus segment (consisting of one-sixteerth of the torus) be:ween stationary TF coils.

The IF cails are a pure tersion D-shape design with a trapezoidal cross secion. The centering forces on the ccils are reccted through a bucking cylinder and an intercoil support structure. 
Table 9.1. TF coil system parameters

\begin{tabular}{|c|c|}
\hline \multirow{2}{*}{\multicolumn{2}{|c|}{ Geometric }} \\
\hline & \\
\hline Number of coils, $\mathrm{N}_{\mathrm{c}}$ & 12 \\
\hline Coil shape & $D$ \\
\hline Horizontal opening, $d_{t f}$ & $6.2 n$ \\
\hline Vertical opening, $h_{t f}$ & $9.8 \mathrm{~m}$ \\
\hline Mean coil circumference, $C$ & $29.5 \mathrm{~m}$ \\
\hline Plasma radius, a & $1.2 \mathrm{~m}$ \\
\hline Major radius, $R$ & $5.0 \mathrm{n}$ \\
\hline \multicolumn{2}{|l|}{ Electromagnetic } \\
\hline Maximum field at winding, $B_{\max }$ & $10.9 \mathrm{~T}$ \\
\hline Field on axis, $B_{T}$ & $5.3 \div$ \\
\hline Total ampere-turns & 132 MAT \\
\hline Stored energy, $W_{c}$ & $18 \mathrm{GJ}$ \\
\hline Number of turns per coil & 733 \\
\hline Structure-to-conductor area ratio & 2.14 \\
\hline Current per turn, $I_{t}$ & $15 \mathrm{kA}$ \\
\hline Conductor & $\begin{array}{l}\mathrm{Nb}_{3} \mathrm{Sr} \text { superconductor with } \\
\text { copper matrix }\end{array}$ \\
\hline $\begin{array}{l}\text { Overall average conductor } \\
\text { current density, JAC }\end{array}$ & $37.5 \mathrm{MA} / \mathrm{m}^{2}$ \\
\hline \multicolumn{2}{|l|}{ Structural } \\
\hline Strain in conductor matrix, $\varepsilon_{m}$ & $<0.2 \%$ \\
\hline Centering force per coil, $F_{c}$ & $470 \times 10^{6} \mathrm{~N}$ \\
\hline Structural material & 300 series stainless steel \\
\hline Weight of each coil, $W_{\text {coil }}$ & $220,000 \mathrm{~kg}$ \\
\hline \multicolumn{2}{|l|}{ Therma 1} \\
\hline Conductor coolant & Supercritical helium \\
\hline $\begin{array}{l}\text { Average helium inlet } \\
\text { temperature, } T_{\text {in }}\end{array}$ & $5.0 \mathrm{~K}$ \\
\hline Helium flow rate/coil, $\mathrm{m}$ & $4 \mathrm{~kg} / \mathrm{sec}$ \\
\hline $\begin{array}{l}\text { Total refrigeration required at } \\
5.0 \mathrm{~K} \text { (includes pumping power), } \mathrm{P}_{\text {ref }}\end{array}$ & $48 \mathrm{~kW}^{\prime}$ \\
\hline
\end{tabular}


The superconducting elements are composed of an insulated, cabled conductor, using $\mathrm{Nb}_{3} \mathrm{Sn}$ filaments in a copper stabilizing matrix. The cabies are encapsulated by a thin stainless steel jacket and cooled by forcec flow supercritical helium, which runs through the interstices of the strands. The conductors are pancake wound around a stainless steel coil suppart bobin. There are eight slots in a bobbin with three $\mathrm{Nb}_{3} \mathrm{Sn}$ superconductors in a slot. The TF coil configuration is shown in Fig. @.l.

ORNL/OWG/FED-79-1258

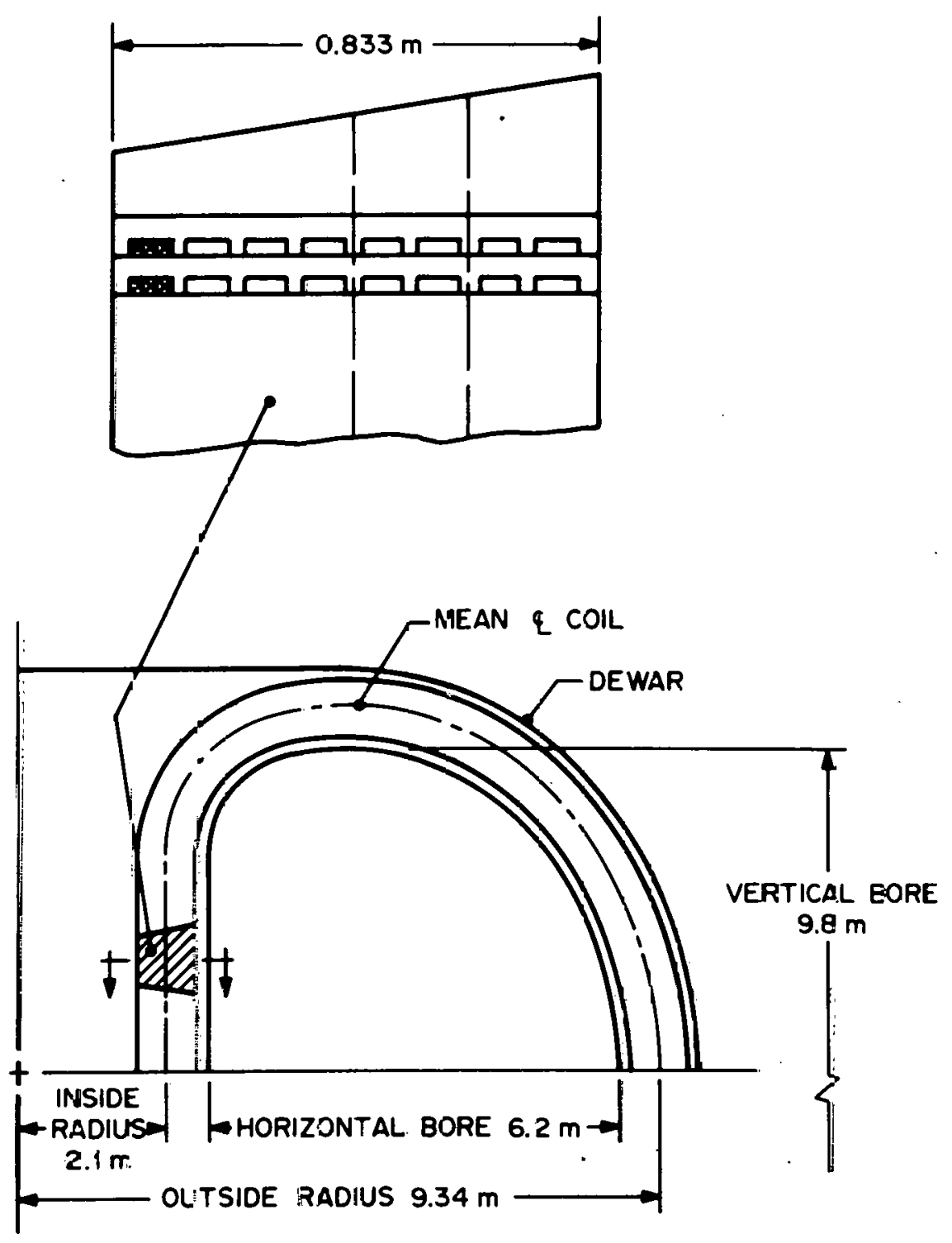

Fig. 9.1. TF coil configuration. 
The superconducting TF coils are designed to be cryostable. The cryogenic recovery capability of the conductor design is defined as the ability to recover to a fully superconducting state from an initially imposed normal zone temperature (driven to that temperature by a sudden heat deposition in the conductor) over a given ncrmal zone length. The present stability requirement ground rule specifies that superconductors shall be able to recover to their fully superconcucting state after $10^{5} \mathrm{~J} / \mathrm{m}^{3}$ of conductor plus helium volume is suddenly deposited in half a turn of the conductor winding. Figure 9.2 indice.tes that the $\mathrm{Nb}_{3} \mathrm{Sn}$ design can meet the requirement with a very small flow rate. However, in order to maintain a turbulent flow in the $\mathrm{Nb}_{3} \mathrm{Sn}$ conductor channels, a flow mass velocity of $2 \mathrm{~g} / \mathrm{sec}-\mathrm{cm}^{2}$ per conductor was selected. At this mass velocity, the stability margin of the $\mathrm{Nb}_{3} \mathrm{Sn}$ superconductors is $>3$. 


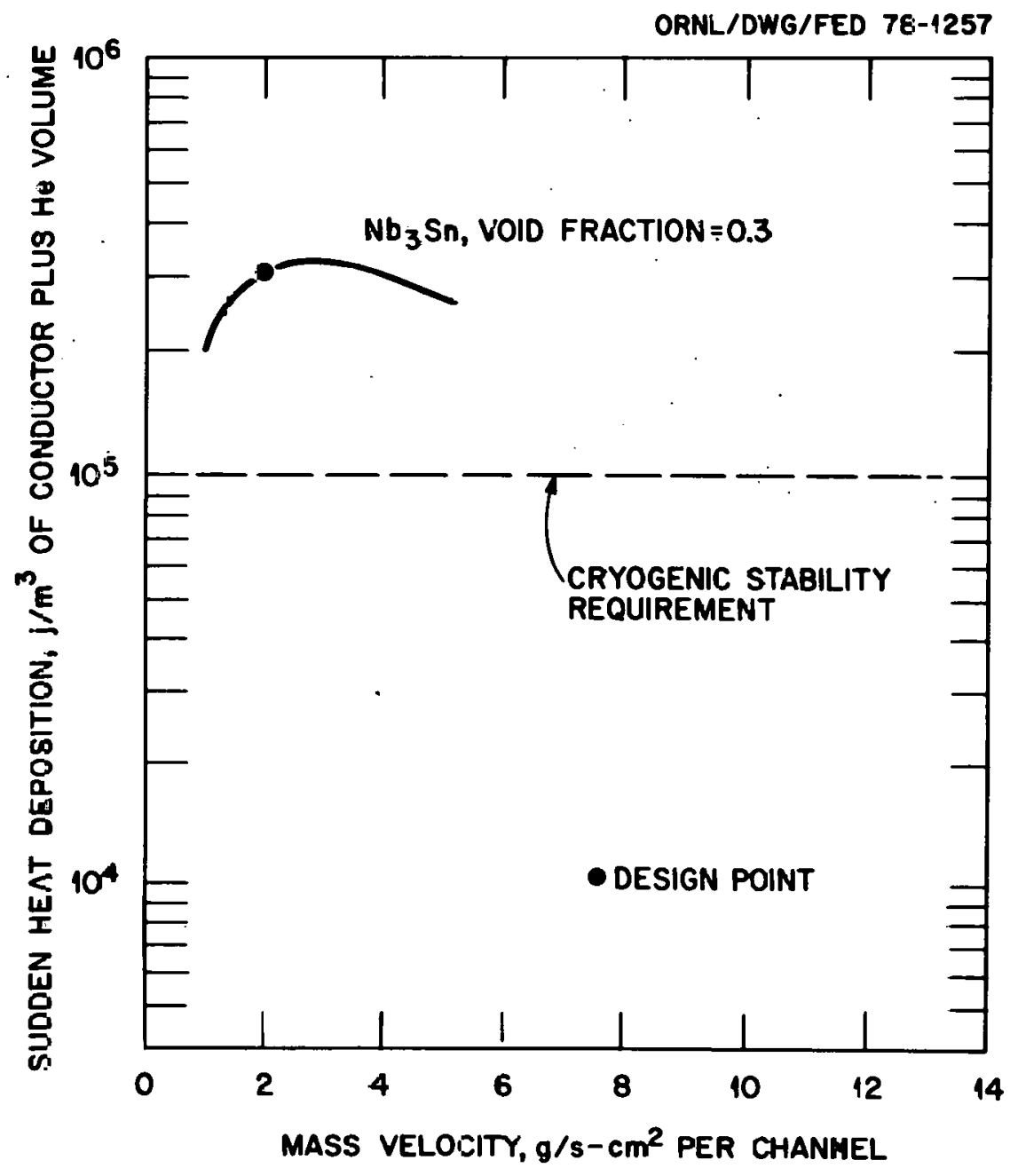

Fig. 7.2. Recovery capability of the TF coil supercondu=tor. 


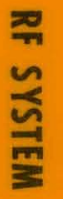




\section{RF SYSTEM}

\subsection{TECHNICAL NEEDS ASSESSMENT}

The use of $r f$ energy to assist during the startup fhase of the tokamak is incorporated into the. TNS Reference Design. The potential advantages of this system include PF system cost reductions and equipment simplification. The major technical need for $r f$ assisted startup appears to be proving that the plasma physics of this approach is faverable (see Sect. 2.2). The key technology issues are discussed below and depicted schematically in Fig. 10.1.

A target power output of $200 \mathrm{~kW}$ per tube appears to be a reasonable objective at this time. It follows that a tube development program should be started for devices operating at $120 \mathrm{GHz}$, using the technical fallout from the Varian Associates 28-Ghz tube development efforts.

The transfer of rf power from the source to the plasma poses several problems in the areas of high power waveguide components and coupling of $\mathrm{rf}$ heating to the plasma. A development/test program should be instituted to investigate the power handling of overs ze waveguides and the design of mode filters. Optimum materials for waveguides and vacuum seals able to resist radiation must also te developed. Some recommended tasks which will provide needed engineering information are outlined below.

\subsubsection{Quasi-Optical Oversize Waveguide}

The characteristics of various oversize circular anc rectangular waveguides should be investigated. Tapers from larger tc smaller and from circular to rectangular guides are of special interest. Breadboard models should be built and used to measure attenuation, voltage standingwave ratio (VSWR), mode characteristics, and the effects of bends, smoothness, material types, and circularity tolerances. Tests would be conducted at low power at a frequency in the $120-3 \mathrm{~Hz}$ range. 


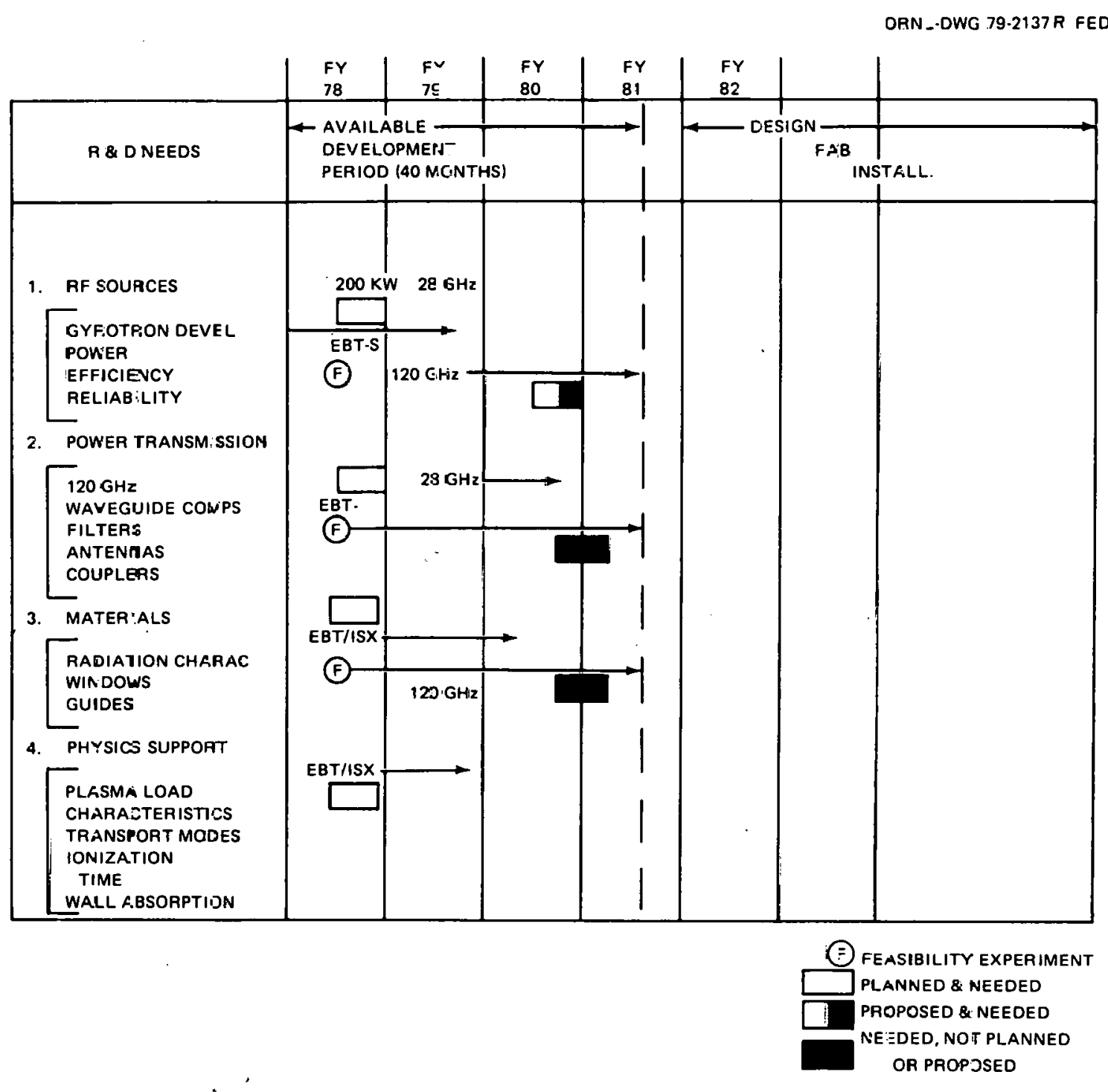

Fig. 10.1. R\&D needs for $r f$ heating system.

Oversize waveguide directional couplers, mode filters, and miter berids should be designed and breadboard models built to measure attenuation, VisWR, and mode purity. Again, tests would be zonducted at low po'ver and $120 \mathrm{GHz}$.

Dnce these investigations are complete, high power sesting of wareguide straight sections, bends, mode filters, and directicnal couplers should take place with $200 \mathrm{~kW}$ at $120 \mathrm{GHz}$ with a $4 \%$ duty cycle, unfer both pressurized and unpressurized conditions. The development of 
the microwave window vacuum seals would tie in with this work (see Sect. 10.1.2).

\subsubsection{Pressurization Window}

Investigations should include evaluation of materials (such as beryllium oxide) for their loss characteristics, power handling, radiation resistance, and strength and evaluation of sossible window locations in the waveguide run. Tests of samples in the waveguide should measure transmission loss and strength by pressurization of the guide at low power. Power handling could be evaluated by subjecting the waveguide and window to $200 \mathrm{~kW}$ (peak) with a $4 \%$ duty cycle, both with and without pressurization. The radiation resistance characseristics should be confirmed either by analysis or through testing.

\subsubsection{Antenna Pattern}

A partial full-scale screen mock-up of the torus could be used to investigate antenna designs and determine those capable of coupling the $r f$ radiation into the plasma. Antenna designs cculd be breadboarded, tested for free space pattern integrity and VSWR, and then mounted on the mock-up for further pattern and VSWR measurements.

\subsection{PROPOSED RF HEATING SYSTEM}

\subsubsection{Function}

The $r$ heating system supplies high power mizrowave energy to the plasma to initiate ionization and heating during startup. The rf assisted startup is expected to significantly reduce $\mathrm{OH}$ power requirements and cost. System parameters are shown in Table 10.1. 
Tajle 10.1. 3F heating system parameters

Structural/configurational

Number of gyroklystrcn tubes

5

Tube length

$1.22 \mathrm{~m}$

Tube diameter

$0.91 \mathrm{~m}$

Performance

Total injiected power

\MW

Pulse length

i. $\mathrm{sec}$

Frequencyi

$120 \mathrm{GHz}$

Injected power per tube $200 \mathrm{~kW}$

Efficiency (gyroklys:ron device)

$30 \%$

Total electrical demand (from grid)

?.5 MVA

\subsubsection{Design Requirements}

The design requiremenis for the $r f$ heating system call for $1.0 \mathrm{MW}$ of injected power delivered at a frequency of $120 \mathrm{GHz}$. Th $\equiv$ pulse length is $2 \mathrm{sec}$ and the duty cycle is $0.4 \%$. The system's lifetime is 10 years.

\subsubsection{Design Description}

The use of microwave heating in the Reference Design is considered to be a promising approach to preionization and may significantily lower the power recuirements and cost of the $\mathrm{OH}$ power supply systems. Preiorization by $r f$ heating can substantially reduce the $\mathrm{OH}$ power supply energy needed for large loop voltage breakdown. Power transmission is accomplished by relatively efficient waveguides. The proposed system is based on a configurction of five $2000-\mathrm{kW}$ gyroklystrons which will deliver 1 NW to the plasma for appraximately $2.0 \mathrm{sec}$.

Five power ampifiers driven by a single master gyrctron oscillatior generate the $\mathrm{rf}$ power. The gyrctron is a new type of microwave vacuum tube jased on the interaction between an electron beam and microwave fields where coupling is achieved by the cyclotron resonance condition. This type of coupling allows the keam and microwave circuit dimensions 
to be large compared to a wavelength. Power de:nsity problems encountered in conventional traveling wave tubes and xlystrons at millimeter wavelengths are not as severe in the gyrotron. Each gyrokiystron amplifier tube has a minimum gain of $30 \mathrm{~dB}$ and produces $200 \mathrm{~kW}$. The single master oscillator operates at the fundamental frequency, produces 1 to $2 \mathrm{~kW}$, and drives the individual power amplifiers through a 5:1 divider network. Each power amplifier tube is $1.22 \mathrm{~m}$ long by $0.92 \mathrm{~m}$ in diameter, requires several electrical power supplies and 011 , water, and fluorocarbon coolants and has a superconducting focusing magnet. The $\mathrm{rf}$ power is transmitted through oversize waveguides. (i.e., the waveguide diameter is approximately 10 times the wavelength) to penetrations in the torus.

Five shielded canisters, each containing a nigh power rf source, are to be mounted on a support platform on top of the tokamak. 
THIS PAGE

WAS INTENTIONALLY

LEFT BLANK 


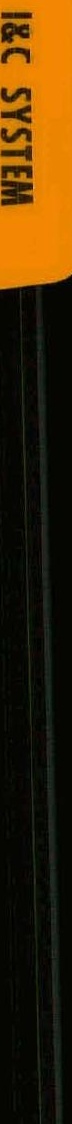




\section{INSTRUMENTATION AND CONTROL S SYSTEM}

\subsection{TECHNICAL NEEDS ASSESSMENT}

The present concepts for the controls and instrumentation of the TNS Reference Design are described in Sect. 11.ć. To däte, there has been little study of the specific controls needs for tokamak reactors, and the projected transient operation of a reactor has only recently been a subject for controls studies. This area needs the attention that would result from an expanding, in-depth investijation into TNS needs and potential solutions. The results from such studies will undoubtedly play a significant role in the early definition of potential problems and the delineation and application of solutions which will shape the transient operation or burn scenario for TNS.

The major controls R\&D requirement to meet the needs of TNS. includes both physics and technology; it may be stated as definition and test of the controls concepts suitable for tokamak operation. The tokamak controls R\&D effort throughout the fusion community has been limited tc very preliminary studies and tests of plasma position control. The major areas of concern (startup, burn, refueling, shutdown, and abort) have hardly been considered. The approach to conirolling a long burn tokamak has only recently been addressed in any significant fashion.

In support of the key technical need discussed above, additional instrumentation and controls R\&D needs include:

(1) Determination of reactor-relevant instrumentation that can adequately survive the projected environment and provide the data for control.

(2) Development of a hybrid computer simulation, bench marked with ISX-B and later machines, to provide a correlated and necessary controls analysis tool.

(3) Assessment of subsidiary systems (those having no direct interface with the plasma system) and of their controls needs, problems, and solutions. These would incluce heat removal and tritium handling systems, power supplies, etc. 
(4) Definition of the diagnostic system approach and equipment matrix to provide the required data handling for experimenters and operators on both a real-time and an archival basis.

11.2 PROPOSED INSTRUMENTATION, CONTROLS, AND DATA HANDLING SYSTEM

\subsubsection{Function}

The instrumentation, contro:s, and data handling (ICDH) system automatically controls the ijkamak plasma and systems through all phases of speration. Semiautonomous operation of subsystem controls and diagnostics is carried out under the supervision of a central computerized conirol. Data from the controls and diagnostics instrumentation are processed and then displayed or stored in an archival system for later on-call display. Fail-safe operations and degraded node options will recaive significant emphasis in the design of the ICDH system.

\subsubsection{Design Fequirements}

The [CDF system will consist of a certra] operations station and several semiautonomous controls substations. The system is centralized in that the machine can be started, operated, and secured from the central operations station and in that the data relevant to machine perfarmance are stored in a central data pool. Upon diract assignment from the central operations station, the semiautonomous controls substations may operate a particular subsystem. However, the responsibility of monitaring all operatiors and the ability to override commands from substations will remain with the central operations station.

Individual subsystem operations, such as testing arid checkout, will be prevented from affecting other elements (operating or dormant) by a system of interlocks and overrides. As the environment permits, each controls substation will be located in proximity to the equifment it monitors and controls to facilitate testing and checkout.

The ICDH system will be constructed with normal industry practices, rodified as necessary for shielding from detrimental effects of nuclear 
radiation and magnetic fields. The equipment $w^{-11}$ incorporate redundancy, diversity, and fail-safe features to minimize maintenance needs. Only monitoring devices and communications links and lines will require remote maintenance. With the exception of certain monitoring devices that may require periodic replacement because of exposure to plasma operation, the system has a design life of 10 years.

\subsubsection{Design Description}

The ICDH system has four major divisions, each with its own monitoring and data handling.

\section{Operations control}

This subsystem controls all machine operations during all phases of the burn cycle and the immediate preparation for the next burn cycle. The supervisory control and the central operatioris station form this division.

\section{Instrumentation and monitoring}

This subsystem measures all pertinent variables and processes the data through appropriate algorithms. The instrumentation used for this normal control and monitoring will use multiplexing microwave and light transmission to facilitate maintenance and to reduce the space needed for penetrations to the machine from the operating stations.

It is desirable to monitor the following parcmeters:

(1) spatial and temporal variations in plasma density,

(2) plasma electron and ion temperatures,

(3) neutron flux at the first wall,

(4) tritium levels at selected locations,

(5) divertor temperatures and coil currents,

(6) poloidal magnetic field strength and coil zurrents,

(7) toroidal magnetic field strength and coil surrents,

(8) neutral beam parameters and status, 
i9) rf hesting system parameters and status, and

(10) fualing rate.

\section{Auxiliaries control}

This sub $\equiv y$ stem controis and manitors the element; that are less frequently conmanded, including the TF power system, the racuum pumping systers, the refuigeration systems, and the electrical susstation.

\section{Abort control}

The abort control subsystem is a fail-safe, scram-tyfe system that prevents machine damage in the event of any expected disruption or operation anomaly. The irstrumentation for this subsystem will receive the stronges: emphasis on reliability and redundancy, and variables related to this area will be continually monitored a: al' stations. 
믈 


\section{INTERNAL DISTRIBUTION}

1. R. G. Alsmiller

2. S. E. Attenberger

3. J. K. Ballou

4. W. R. Becraft

5. L. A. Berry

6. E. E. Bloom

7. A. L. Boch

8. T. G. Brown

9. E. H. Bryant

10. J. D. Callen

11. D. D. Cannon

12. R. J. Colchin

13. R. A. Dory

14. J. L. Dunlap

15. P. N. Haubenreich

16. J. T. Hogan

17. W. A. Houlberg

18. T. J. Huxford

19. M. S. Lubell

20. J. W. Lue

21. A. T. Mense

22. 0. B. Morgan

23. L. W. Nelms

24. Y-K. M. Peng
25. H. Postma

26. R. L. Reid

27. J. A. Rome

28. M. W. Rosenthal

29. R. T. Santoro

30. C. Sardella

31. J. L. Scott

32. T. E. Shannon

33. J. Sheffield

34-85. D. Steiner

86. N. A. Uckan

87. J. S. Watson

88. W. M. Wells

89. F. W. Wiffen

90. G. W. Wiseman

91. H. T. Yeh

92-93. Central Research Library

94-95. Fusion Energy Division Library

96. Fusion Energy Division Communications Center

97-98. Laboratory Records Department

99. Laboratory Records, ORNL-RC

100. ORNL Patent Office

101. Document Reference Section

\section{EXTERNAL DISTRIBUTION}

102. R. E. Aronstein, Bechtel, P.0. Box 3965, San Francisco, CA 94119

103. D. J. Anthony, General Electric Co., B1dg. 23, Rm. 290, 1 River Rd., Schenectady, NY 12345

104. J. E. Baublitz, Office of Fusion Energy, Department of Energy, Washington, DC 20545

105. D. S. Beard, Office of Fusion Energy, Department of Energy, Washington, DC 20545

106. G. Benedict, Deaprtment of Energy, Oak Ridge Operations, P.0. Box E, Oak Ridge, TN 37830

107. S. L. Bogart, Science Applications Inc., 8400 Westpark Drive, McLean, VA 22102

108. Roger Boom, University of Wisconsin, Madison, WI 53706

109. R. Botwin, Grumman Aerospace Corp., Bethpage, NY 11714

110. R. N. Cherdack, Burns \& Roe, Inc., 283 Highway 17, Paramus, Nu 07652 
111. J. F. Clarke, Office of Fusion Energy, Department of Energy, Washington, DC 20545

112. F. E. Coffman, Office of Fusion Energy, Department of Energy, Washington, DC 20545

113. D. Cohn, Massachusetts Institute of Technology, Cambridge, MA 02139

114. J. W. Coursen, Grumman Aerospace Corp., Bethpage, NY 11714

115. J. G. Crocker, EG\&G Idaho, Idaho National Engineering Laboratory, P.0. Box 1625, Idaho Falls, ID 83401

116. Library, Culham Laboratory, Abingdon, Oxon, 0x14 3DB, United Kingdom

117. N. Anne Davies, Office of Fusion Energy, Department of Energy, Washington, DC 20545

118. H. W. Deckman, Advanced Energy Systems Laboratory, Government Research Laboratories. Exxon Researr.h and Fngineering Co., P.0. Box 8, Linden, NJ 07036

119. A. Favale, Grumman Aerospace Corporation, Bethpage, NY 11714

120. J. J. Ferrante, Large Coil Program, Bldg. 2-708, General Electric, Co., 1 River Rd., Schenectady, NY 12345

121. F. Fickett, National Bureau of Standards, Boulder, CO 80302

122. C. A. Flanagan, Westinghouse Electric Corp., Fusion Power Systems, P.0. Box 10864, Pittsburgh, PA 15236

123. H. K. Forsen, Exxon Nuclear Co., Inc., 777 106th Ave., Bellevue, WA 98009

124. J. W. French, EBASCO Services, Inc., Princeton University, P.0. Box 451, Princeton, NJ 08540

125. G. M. Fuller, McDonnell-Douglas, Dept. Ë-450, Bìdg. 10613, Rm. 370, St. Louts, MO 63166

126. H. P. Furth, Princeton Plasma Physics Laboratory, Princeton IIniversity, P.0. Box 451 , Princeton, NJ 08540

127. A. Gaines, Combustion Engineering, 100 Prospect Hill Rd., Windsor, CT 06095

128. A. Gibson, Culham Laboratory, Abingdon, 0xon, 0X14 3DB, United Kingdom

129. R. W. Gould, Mail Stop 116-81, California Institute of Technology, Pasadena, CA 91125

130. E. Gregory, Airco Inc., Murray Hill, NJ 07974

131. D. S. Hackley, Large Coil Program, General Dynamics-Convair Division, P.0. Box 80847, Sall Dieyu, CA 92138

132. R. Hancox, Culham Laboratory, Abingdon, Oxon, 0X74 3DB, United Kingdom

133. C. R. Head, Office of Fusion Energy, Department of Energy, Washington, DC 20545

134. C. Henning, Lawrence Livermore Laboratory, P.0. Box 808, Livermore, CA 94550

135. R. L. Hirsch, Exxon Research and Engineering Co., P.0. Box 101 , Florham Park, NJ 07932

136. Anthony Hsu, Office of Fusion Energy, Department of Energy, Washington, DC 20545

137. D. L. Jassby, Princeton Plasma Physics Laboratory, Princeton University, P.0. Box 451, Princeton, NJ 08540 
138. D. L. Kummer, McDonnel1-Douglas Astronautics Co., East, P.0. Box 516, St. Louis, MO 63766

139. D. G. McAlees, Manager, ETF Systems Interface, Exxon Nuclear Co., Inc., 777 106th Avenue, N.E., Bellevue, WA 98009

140. V. A. Maroni, Argonne National Laboratory, 9700 South Cass Ave., Argonne, IL 60439

141. D. M. Meade, Princeton Plasma Physics Laboratory, Princeton University, P.0. Box 451, Princeton, NJ 08540

142. R, L. Miller, General Atomic Co., P.0. Box 81608, San Diego, CA 92138

143. M. R. Murphy, Office of Fusion Energy, Department of Energy, Washington, DC 20545

144. J. G. Murray, Princeton Plasma Physics Laboratory, Princeton University, P.0. Box 451, Princeton, NJ 08540

145. L. K. Price, Department of Energy, Oak Ridge Operations, P.0. Box E, Oak Ridge, TN 37830

146. J. M. Rawls, General Atomic Co., P.0. Box 81608, San Diego, CA 92138

147. P. Reardon, Princeton Plasma Physics Laboratory, Princeton University, P.0. Box 451, Princeton, NJ 08540

148. T. Reuther, Office of Fusion Energy, Department of Energy, Washington, DC 20545

149. M. Roberts, Office of Fusion Energy, Department of Energy, Washington, DC 20545

150. D. J. Rose, Department of Nuclear Engineering, Massachusetts Institute of Technology, Cambridge, MA 02139

151. M. N. Rosenbluth, School of Natural Sciences, Princeton University, P.0. Box 451, Princeton, NJ 08540

152. C. Rosner, Intermagnetics General Corp., Charles Industrial Park, New Karner Rd., Guilderland, NY 12084

153. P. H. Sager, Jr., General Atomic Co.., P.0. Box 81608, San Diego, CA 92138

154. G. Schllling, Princetón Plàsma Physics Laboratory, Princeton University, P.0. Box 451 , Princeton, NJ 08540

155. Z. M. Shapiro, Westinghouse Electric Corp., Fusion Power Systems Department, P.0. Box 10864, Pittsburgh, PA 15236

156. G. Siegel, Tennessee Vally Authority, 1360 Commerce Union Bank Bldg., Chattanooga, TN 37401

157. A. Simon, University of Rochester, Rochester, NY 14627

158. W. M. Stacey, Jr., School of Nuclear Engineering, Georgia Institute of Technology, Atlanta, GA 30332

159. J. Stekly, Magnetic Corp. of America, 179 Bear Hill Rd., Wal tham, MA 02154

160. L. D. Stewart, Princeton Plasma Physics Laboratory, Princeton University, P.0. Box 451, Princeton, NJ 08540

161. C. Taylor, Controlled Thermonuclear Research, Mail Code L-382, Lawrence Livermore Laboratory, P.0. Box 808, Livermore, CA 94550

162. F. Thomas, Grumman Aerospace Corp., Bethpage, NY 11714

163. T. C. Varljen, Westinghouse Electric Corp., Fusion Power Systems, P.0. Box 10864, Pittsburgh, PA 15236 
164. S. S. Waddle, Department of Energy, Oak Ridge Operations, P.O. Box E, Oak Ridge, TN 37830

165. J. Willis, Office of Fusion Energy, Department of Energy, Washington, DC 20545

166. W. Wilkes, Mound Laboratories, Miamisburg, $\mathrm{OH} 45432$

167. H. H. Woodson, Department of Electrical Engineering, University of Texas, Austin, TX 78712

168. W. W. Withee, Energy Systems, General Dynamics-Convair Division, P.0. Box 80847, San Diego, CA 92138

169. S. Yoshikawa, Princeton Plasma Physics Laboratory, Princeton University, P.0. Box 451, Princeton, NJ 08540

170. J. L. Young, Large Coil Program, Westinghouse Electric Corp., 1310 Beulah Road, Pittsburgh, PA 15235

171. E. Ziurys, Office of Fusion Energy, Department of Energy, Washington, DC 20545

172. K. Zwilsky, Office of Fusion Energy; Department of Energy, Washington, DC 20545

173. Office of Assistant Manager, Energy Research and Development, Department of Energy, Oak Ridge Operations, P.O. Box E, Oak Ridge, TN 37830

174-324. Given distribution as shown in TID-4500, Magnetic Fusion Energy (Distribution Category UC-20 d, Fusion Systems) 